\title{
Additional Recursion Relations, Factorizations, and Diophantine Properties Associated with the Polynomials of the Askey Scheme
}

\author{
M. Bruschi, ${ }^{1,2}$ F. Calogero, ${ }^{1,2}$ and R. Droghei ${ }^{3}$ \\ ${ }^{1}$ Dipartimento di Fisica, Università di Roma "La Sapienza", 00185 Roma, Italy \\ ${ }^{2}$ Istituto Nazionale di Fisica Nucleare, Sezione di Roma, 00185 Roma, Italy \\ ${ }^{3}$ Dipartimento di Fisica, Università Roma Tre, 00146 Roma, Italy \\ Correspondence should be addressed to F. Calogero, francesco.calogero@roma1.infn.it
}

Received 29 July 2008; Accepted 1 December 2008

Recommended by M. Lakshmanan

In this paper, we apply to (almost) all the "named" polynomials of the Askey scheme, as defined by their standard three-term recursion relations, the machinery developed in previous papers. For each of these polynomials we identify at least one additional recursion relation involving a shift in some of the parameters they feature, and for several of these polynomials characterized by special values of their parameters, factorizations are identified yielding some or all of their zerosgenerally given by simple expressions in terms of integers (Diophantine relations). The factorization findings generally are applicable for values of the Askey polynomials that extend beyond those for which the standard orthogonality relations hold. Most of these results are not (yet) reported in the standard compilations.

Copyright (c) 2009 M. Bruschi et al. This is an open access article distributed under the Creative Commons Attribution License, which permits unrestricted use, distribution, and reproduction in any medium, provided the original work is properly cited.

\section{Introduction}

Recently Diophantine findings and conjectures concerning the eigenvalues of certain tridiagonal matrices, and correspondingly the zeros of the polynomials associated with their secular equations, were arrived at via the study of the behavior of certain isochronous manybody problems of Toda type in the neighborhood of their equilibria [1,2] (for a review of these and other analogous results, see [3, Appendix C]). To prove (some of) these conjectures a theoretical framework was then developed [4-6], involving polynomials defined by threeterm recursion relations-hence being, at least for appropriate ranges of the parameters they feature, orthogonal. (This result is generally referred to as "Favard theorem," on the basis of [7]; however, as noted by Ismail, a more appropriate name is "spectral theorem for orthogonal polynomials" [8]). Specific conditions were identified-to be satisfied by the 
coefficients, featuring a parameter $v$, of these recursion relations-sufficient to guarantee that the corresponding polynomials also satisfy a second three-term recursion relation involving shifts in that parameter $v$; and via this second recursion relation, Diophantine results of the kind indicated above were obtained [5]. In Section 2, in order to make this paper essentially self-contained, these developments are tersely reviewed-and also marginally extended, with the corresponding proofs relegated to an appendix to avoid interrupting the flow of the presentation. We then apply, in Section 3, this theoretical machinery to the "named" polynomials of the Askey scheme [9], as defined by the basic three-term recursion relation they satisfy: this entails the identification of the parameter $v$-which can often be done in more than one way, especially for the named polynomials involving several parametersand yields the identification of additional recursion relations satisfied by (most of) these polynomials. Presumably such results (especially after they have been discovered) could also be obtained by other routes-for instance, by exploiting the relations of these polynomials with hypergeometric functions: we did not find them (except in some very classical cases) in the standard compilations [9-13], where they in our opinion deserve to be eventually recorded. Moreover, our machinery yields factorizations of certain of these polynomials entailing the identification of some or all of their zeros, as well as factorizations relating some of these polynomials (with different parameters) to each other. Again, most of these results seem new and deserving to be eventually recorded in the standard compilations although they generally require that the parameters of the named polynomials do not satisfy the standard restrictions required for the orthogonality property. To clarify this restriction let us remark that an elementary example of such factorizations-which might be considered the prototype of formulas reported below for many of the polynomials of the Askey schemereads as follows:

$$
L_{n}^{(-n)}(x)=\frac{(-x)^{n}}{n !}, \quad n=0,1,2, \ldots
$$

where $L_{n}^{(\alpha)}(x)$ is the standard (generalized) Laguerre polynomial of order $n$, for whose orthogonality,

$$
\int_{0}^{\infty} d x x^{\alpha} \exp (-x) L_{n}^{(\alpha)}(x) L_{m}^{(\alpha)}(x)=\delta_{n m} \frac{\Gamma(n+\alpha+1)}{n !}
$$

it is, however, generally required that $\operatorname{Re} \alpha>-1$. This formula, (1.1a), is well known and it is indeed displayed in some of the standard compilations reporting results for classical orthogonal polynomials (see, e.g., page 109 of the classical book by Magnus and Oberhettinger [14] or [11, Equation 8.973.4]). And this remark applies as well to the following neat generalization of this formula, reading

$$
L_{n}^{(-m)}(x)=(-1)^{m} \frac{(n-m) !}{n !} x^{m} L_{n-m}^{(m)}(x), \quad m=0,1, \ldots, n, n=0,1,2, \ldots
$$

which qualifies as well as the prototype of formulas reported below for many of the polynomials of the Askey scheme. (Note, incidentally, that this formula can be inserted without difficulty in the standard orthogonality relation for generalized Laguerre polynomials, (1.1b), reproducing the standard relation: the singularity of the weight function gets indeed neatly 
compensated by the term $x^{m}$ appearing in the right-hand side of (1.1c). Presumably, this property - and the analogous version for Jacobi polynomials-is well known to most experts on orthogonal polynomials; e.g., a referee of this paper wrote "Although I have known of (1.1c) for a long time, I have neither written it down nor saw it stated explicitly. It is clear from reading [15, Paragraph 6.72] that Szëgo was aware of (1.1c) and the more general case of Jacobi polynomials.") Most of the formulas (analogous to (1.1c) and (1.1a)) for the named polynomials of the Askey scheme that are reported below are instead, to the best of our knowledge, new: they do not appear in the standard compilations where we suggest they should be eventually recorded, in view of their neatness and their Diophantine character. They could of course be as well obtained by other routes than those we followed to identify and prove them (it is indeed generally the case that formulas involving special functions, after they have been discovered, are easily proven via several different routes). Let us however emphasize that although the results reported below have been obtained by a rather systematic application of our approach to all the polynomials of the Askey scheme, we do not claim that the results reported exhaust all those of this kind featured by these polynomials. And let us also note that, as it is generally done in the standard treatments of "named" polynomials [9-13], we have treated separately each of the differently "named" classes of these polynomials, even though "in principle" it would be sufficient to only treat the most general class of them-Wilson polynomials-that encompasses all the other classes via appropriate assignments (including limiting ones) of the 4 parameters it features. Section 4 mentions tersely possible future developments.

\section{Preliminaries and Notation}

In this section we report tersely the key points of our approach, mainly in order to make this paper self-contained-as indicated above-and also to establish its notation: previously known results are of course reported without their proofs, except for an extension of these findings whose proof is relegated to Appendix A.

Hereafter we consider classes of monic polynomials $p_{n}^{(v)}(x)$, of degree $n$ in their argument $x$ and depending on a parameter $v$, defined by the three-term recursion relation:

$$
p_{n+1}^{(v)}(x)=\left(x+a_{n}^{(v)}\right) p_{n}^{(v)}(x)+b_{n}^{(v)} p_{n-1}^{(v)}(x)
$$

with the "initial" assignments

$$
p_{-1}^{(v)}(x)=0, \quad p_{0}^{(v)}(x)=1
$$

clearly entailing

$$
p_{1}^{(v)}(x)=x+a_{0}^{(v)}, \quad p_{2}^{(v)}(x)=\left(x+a_{1}^{(v)}\right)\left(x+a_{0}^{(v)}\right)+b_{1}^{(v)}
$$

and so on. (In some cases the left-hand side of the first (2.1b) might preferably be replaced by $b_{0}^{(v)} p_{-1}^{(v)}(x)$, to take account of possible indeterminacies of $b_{0}^{(v)}$.) 
Notation. Here and hereafter the index $n$ is a nonnegative integer (but some of the formulas written below might make little sense for $n=0$, requiring a-generally quite obviousspecial interpretation), and $a_{n}^{(v)}, b_{n}^{(v)}$ are functions of this index $n$ and of the parameter $v$. They might-indeed they often do-also depend on other parameters besides $v$ (see below); but this parameter $v$ plays a crucial role, indeed the results reported below emerge from the identification of special values of it (generally simply related to the index $n$ ).

Let us recall that the theorem which guarantees that these polynomials, being defined by the three-term recursion relation (2.1), are orthogonal (with a positive definite, albeit a priori unknown, weight function), requires that the coefficients $a_{n}^{(v)}$ and $b_{n}^{(v)}$ be real and that the latter be negative, $b_{n}^{(v)}<0$ (see, e.g., [16]).

\subsection{Additional Recursion Relation}

Proposition 2.1. If the quantities $A_{n}^{(v)}$ and $\omega^{(v)}$ satisfy the nonlinear recursion relation

$$
\left[A_{n-1}^{(v)}-A_{n-1}^{(v-1)}\right]\left[A_{n}^{(v)}-A_{n-1}^{(v-1)}+\omega^{(v)}\right]=\left[A_{n-1}^{(v-1)}-A_{n-1}^{(v-2)}\right]\left[A_{n-1}^{(v-1)}-A_{n-2}^{(v-2)}+\omega^{(v-1)}\right]
$$

with the boundary condition

$$
A_{0}^{(v)}=0
$$

(where, without significant loss of generality, this constant is set to zero rather than to an arbitrary $v$-independent value A: see [5, Equation (4a)]; and we also replaced, for notational convenience, the quantity $\alpha^{(v)}$ previously used [5] with $\left.\omega^{(v)}\right)$, and if the coefficients $a_{n}^{(v)}$ and $b_{n}^{(v)}$ are defined in terms of these quantities by the following formulas:

$$
\begin{gathered}
a_{n}^{(v)}=A_{n+1}^{(v)}-A_{n}^{(v)}, \\
b_{n}^{(v)}=\left[A_{n}^{(v)}-A_{n}^{(v-1)}\right]\left[A_{n}^{(v)}-A_{n-1}^{(v-1)}+\omega^{(v)}\right],
\end{gathered}
$$

then the polynomials $p_{n}^{(v)}(x)$ identified by the recursion relation (2.1) satisfy the following additional recursion relation (involving a shift both in the order $n$ of the polynomials and in the parameter $v$ ):

$$
p_{n}^{(v)}(x)=p_{n}^{(v-1)}(x)+g_{n}^{(v)} p_{n-1}^{(v-1)}(x)
$$

with

$$
g_{n}^{(v)}=A_{n}^{(v)}-A_{n}^{(v-1)}
$$

This proposition corresponds to [5, Proposition 2.3]. (As suggested by a referee, let us also mention that recursions in a parameter-albeit of a very special type and different from that reported above—were also presented long ago in a paper by Dickinson et al. [17].) 
Alternative conditions sufficient for the validity of Proposition 2.1 and characterizing directly the coefficients $a_{n}^{(v)}, b_{n}^{(v)}$, and $g_{n}^{(v)}$ read as follows (see [5, Appendix B]):

$$
\begin{gathered}
a_{n}^{(v)}-a_{n}^{(v-1)}=g_{n+1}^{(v)}-g_{n}^{(v)}, \\
b_{n-1}^{(v-1)} g_{n}^{(v)}-b_{n}^{(v)} g_{n-1}^{(v)}=0,
\end{gathered}
$$

with

$$
g_{n}^{(v)}=-\frac{b_{n}^{(v)}-b_{n}^{(v-1)}}{a_{n}^{(v)}-a_{n-1}^{(v-1)}},
$$

and the "initial" condition

$$
g_{1}^{(v)}=a_{0}^{(v)}-a_{0}^{(v-1)}
$$

entailing via (2.5c) (with $n=1$ )

$$
b_{1}^{(v)}-b_{1}^{(v-1)}+\left(a_{0}^{(v)}-a_{0}^{(v-1)}\right)\left(a_{1}^{(v)}-a_{0}^{(v-1)}\right)=0
$$

and via (2.5a) (with $n=0)$

$$
g_{0}^{(v)}=0
$$

Proposition 2.2. Assume that the class of (monic, orthogonal) polynomials $p_{n}^{(v)}(x)$ defined by the recursion (2.1) satisfies Proposition 2.1, hence that they also obey the ("second") recursion relation (2.4). Then, there also holds the relations:

$$
\begin{gathered}
p_{n}^{(v)}(x)=\left[x-x_{n}^{(1, v)}\right] p_{n-1}^{(v-1)}(x)+b_{n-1}^{(v-1)} p_{n-2}^{(v-1)}(x), \\
x_{n}^{(1, v)}=-\left[a_{n-1}^{(v-1)}+g_{n}^{(v)}\right]
\end{gathered}
$$

in addition to

$$
\begin{gathered}
p_{n}^{(v)}(x)=\left[x-x_{n}^{(2, v)}\right] p_{n-1}^{(v-2)}(x)+c_{n}^{(v)} p_{n-2}^{(v-2)}(x), \\
x_{n}^{(2, v)}=-\left[a_{n-1}^{(v-2)}+g_{n}^{(v)}+g_{n}^{(v-1)}\right], \\
c_{n}^{(v)}=b_{n-1}^{(v-2)}+g_{n}^{(v)} g_{n-1}^{(v-1)},
\end{gathered}
$$


as well as

$$
\begin{gathered}
p_{n}^{(v)}(x)=\left[x-x_{n}^{(3, v)}\right] p_{n-1}^{(v-3)}(x)+d_{n}^{(v)} p_{n-2}^{(v-3)}(x)+e_{n}^{(v)} p_{n-3}^{(v-3)}(x), \\
x_{n}^{(3, v)}=-\left[a_{n-1}^{(v-3)}+g_{n}^{(v)}+g_{n}^{(v-1)}+g_{n}^{(v-2)}\right], \\
d_{n}^{(v)}=b_{n-1}^{(v-3)}+g_{n}^{(v)} g_{n-1}^{(v-2)}+g_{n}^{(v-1)} g_{n-1}^{(v-2)}+g_{n}^{(v)} g_{n-1}^{(v-1)}, \\
e_{n}^{(v)}=g_{n}^{(v)} g_{n-1}^{(v-1)} g_{n-2}^{(v-2)} .
\end{gathered}
$$

These findings correspond to [6, Proposition 1].

\subsection{Factorizations}

In the following we introduce a second parameter $\mu$, but for notational simplicity we do not emphasize explicitly the dependence of the various quantities on this parameter.

Proposition 2.3. If the (monic, orthogonal) polynomials $p_{n}^{(v)}(x)$ are defined by the recursion relation (2.1) and the coefficients $b_{n}^{(v)}$ satisfy the relation

$$
b_{n}^{(n+\mu)}=0,
$$

entailing that for $v=n+\mu$, the recursion relation (2.1a) reads

$$
p_{n+1}^{(n+\mu)}(x)=\left(x+a_{n}^{(n+\mu)}\right) p_{n}^{(n+\mu)}(x)
$$

then there holds the factorization

$$
p_{n}^{(m+\mu)}(x)=\widetilde{p}_{n-m}^{(-m)}(x) p_{m}^{(m+\mu)}(x), \quad m=0,1, \ldots, n,
$$

with the "complementary" polynomials $\tilde{p}_{n}^{(-m)}(x)$ (of course of degree $n$ ) defined by the following threeterm recursion relation analogous (but not identical) to (2.1):

$$
\begin{gathered}
\tilde{p}_{n+1}^{(-m)}(x)=\left(x+a_{n+m}^{(m+\mu)}\right) \tilde{p}_{n}^{(-m)}(x)+b_{n+m}^{(m+\mu)} \tilde{p}_{n-1}^{(-m)}(x), \\
\tilde{p}_{-1}^{(-m)}(x)=0, \quad \tilde{p}_{0}^{(-m)}(x)=1,
\end{gathered}
$$

entailing

$$
\begin{gathered}
\tilde{p}_{1}^{(-m)}(x)=x+a_{m}^{(m+\mu)}, \\
\tilde{p}_{2}^{(-m)}(x)=\left(x+a_{m+1}^{(m+\mu)}\right)\left(x+a_{m}^{(m+\mu)}\right)+b_{m+1}^{(m+\mu)}=\left(x-x_{m}^{(+)}\right)\left(x-x_{m}^{(-)}\right)
\end{gathered}
$$


with

$$
x_{m}^{( \pm)}=\frac{1}{2}\left\{-a_{m}^{(m+\mu)}-a_{m+1}^{(m+\mu)} \pm\left[\left(a_{m}^{(m+\mu)}-a_{m+1}^{(m+\mu)}\right)^{2}-4 b_{m+1}^{(m+\mu)}\right]^{1 / 2}\right\}
$$

and so on.

This is a slight generalization (proven below, in Appendix A) of [5, Proposition 2.4]. Note incidentally that also the complementary polynomials $\tilde{p}_{n}^{(-m)}(x)$, being defined by threeterms recursion relations, see (2.12a), may belong to orthogonal families, hence they should have to be eventually investigated in such a context, perhaps applying also to them the kind of findings reported in this paper.

The following two results are immediate consequences of Proposition 2.3.

Corollary 2.4. If (2.9) holds—entailing (2.10) and (2.11) with (2.12)—the polynomial $p_{n}^{(n-1)}(x)$ has the zero $-a_{n-1}^{(n-1)}$,

$$
p_{n}^{(n-1+\mu)}\left(-a_{n-1}^{(n-1+\mu)}\right)=0
$$

and the polynomial $p_{n}^{(n-2+\mu)}(x)$ has the two zeros $x_{n-2}^{( \pm)}$(see $\left.(2.12 \mathrm{e})\right)$,

$$
p_{n}^{(n-2+\mu)}\left(x_{n-2}^{( \pm)}\right)=0
$$

The first of these results is a trivial consequence of (2.10); the second is evident from (2.11) and (2.12d). Note, moreover, that from the factorization formula (2.11), one can likewise find explicitly 3 zeros of $p_{n}^{(n-3+\mu)}(x)$ and 4 zeros of $p_{n}^{(n-4+\mu)}(x)$, by evaluation from $(2.12) \tilde{p}_{3}^{(-m)}(x)$ and $\tilde{p}_{4}^{(-m)}(x)$ and by taking advantage of the explicit solvability of algebraic equations of degrees 3 and 4.

These findings often have a Diophantine connotation, due to the neat expressions of the zeros $-a_{n-1}^{(n-1+\mu)}$ and $x_{n-2}^{( \pm)}$in terms of integers.

Corollary 2.5. If (2.9) holds—entailing (2.10) and (2.11) with (2.12)—and moreover the quantities $a_{n}^{(m)}$ and $b_{n}^{(m)}$ satisfy the properties

$$
a_{n-m}^{(-m+\mu)}(\underline{\rho})=a_{n}^{(m+\tilde{\mu})}(\underline{\tilde{\rho}}), \quad b_{n-m}^{(-m+\mu)}(\underline{\rho})=b_{n}^{(m+\tilde{\mu})}(\underline{\tilde{\rho}})
$$

then clearly

$$
\tilde{p}_{n}^{(m)}(x ; \underline{\rho})=p_{n}^{(m+\tilde{\mu})}(x ; \underline{\tilde{\rho}})
$$

entailing that the factorization (2.11) takes the neat form

$$
p_{n}^{(m+\mu)}(x ; \underline{\rho})=p_{n-m}^{(-m+\tilde{\mu})}(x ; \underline{\tilde{\rho}}) p_{m}^{(m+\mu)}(x ; \underline{\rho}), \quad m=0,1, \ldots, n .
$$


Note that-for future convenience, see below-one has emphasized explicitly the possibility that the polynomials depend on additional parameters (indicated with the vector variables $\underline{\rho}$, resp., $\underline{\tilde{\rho}}$; these additional parameters must of course be independent of $n$, but they might depend on $\bar{m}$ ).

The following remark is relevant when both Propositions 2.1 and 2.2 hold.

Remark 2.6. As implied by (2.3b), the condition (2.9) can be enforced via the assignment

$$
\omega^{(v)}=A_{v-1}^{(v-1+\mu)}-A_{v}^{(v+\mu)}
$$

entailing that the nonlinear recursion relation (2.3a) reads

$$
\begin{aligned}
& {\left[A_{n-1}^{(v)}-A_{n-1}^{(v-1)}\right]\left[A_{n}^{(v)}-A_{n-1}^{(v-1)}+A_{v-1}^{(v-1+\mu)}-A_{v}^{(v+\mu)}\right]} \\
& \quad=\left[A_{n-1}^{(v-1)}-A_{n-1}^{(v-2)}\right]\left[A_{n-1}^{(v-1)}-A_{n-2}^{(v-2)}+A_{v-2}^{(v-2+\mu)}-A_{v-1}^{(v-1+\mu)}\right] .
\end{aligned}
$$

Corollaries 2.4 and 2.5 and Remark 2.6 are analogous to [5, Corollaries 2.5 and 2.6 and Remark 2.7].

\subsection{Complete Factorizations and Diophantine Findings}

The Diophantine character of the findings reported below is due to the generally neat expressions of the following zeros in terms of integers (see in particular the examples in Section 3).

Proposition 2.7. If the (monic, orthogonal) polynomials $p_{n}^{(v)}(x)$ are defined by the three-term recursion relations (2.1) with coefficients $a_{n}^{(v)}$ and $b_{n}^{(v)}$ satisfying the requirements sufficient for the validity of both Propositions 2.1 and 2.2 (namely (2.3), with (2.2) and (2.9), or just with (2.18)), then

$$
p_{n}^{(n+\mu)}(x)=\prod_{m=1}^{n}\left[x-x_{m}^{(1, m+\mu)}\right]
$$

with the expressions (2.6b) of the zeros $x_{m}^{(1, v)}$ and the standard convention according to which a product equals unity when its lower limit exceeds its upper limit. Note that these $n$ zeros are nindependent (except for their number). In particular,

$$
p_{0}^{(\mu)}(x)=1, \quad p_{1}^{(1+\mu)}(x)=x-x_{1}^{(1,1+\mu)}, \quad p_{2}^{(2+\mu)}(x)=\left[x-x_{1}^{(1,2+\mu)}\right]\left[x-x_{2}^{(1,2+\mu)}\right],
$$

and so on.

These findings correspond to [6, Proposition 2.2 (first part)].

The following results are immediate consequences of Proposition 2.7 and of Corollary 2.4. 
Corollary 2.8. If Proposition 2.7 holds, then also the polynomials $p_{n}^{(n-1+\mu)}(x)$ and $p_{n}^{(n-2+\mu)}(x)$ (in addition to $p_{n}^{(n+\mu)}(x)$, see (2.19)) can be written in the following completely factorized form (see (2.6b) and $(2.12 \mathrm{e}))$ :

$$
\begin{gathered}
p_{n}^{(n-1+\mu)}(x)=\left[x+a_{n-1}^{(n-1)}\right] \prod_{m=1}^{n-1}\left[x-x_{m}^{(1, m+\mu)}\right], \\
p_{n}^{(n-2+\mu)}(x)=\left[x-x_{m}^{(+)}\right]\left[x-x_{m}^{(-)}\right] \prod_{m=1}^{n-2}\left[x-x_{m}^{(1, m+\mu)}\right] .
\end{gathered}
$$

Analogously, complete factorizations can clearly be written for the polynomials $p_{n}^{(n-3+\mu)}(x)$ and $p_{n}^{(n-4+\mu)}(x)$, see the last part of Corollary 2.4.

And of course the factorization (2.11) together with (2.19a) entails the (generally Diophantine) finding that the polynomial $p_{n}^{(m+\mu)}(x)$ with $m=1, \ldots, n$ features the $m$ zeros $x_{\ell}^{(1, \ell+\mu)}, \ell=1, \ldots, m$, see (2.6b):

$$
p_{n}^{(m+\mu)}\left(x_{\ell}^{(1, \ell+\mu)}\right)=0, \quad \ell=1, \ldots, m, m=1, \ldots, n .
$$

Proposition 2.9. Assume that, for the class of polynomials $p_{n}^{(v)}(x)$, there hold the preceding Proposition 2.1, and moreover that, for some value of the parameter $\mu$ (and of course for all nonnegative integer values of $n$ ), the coefficients $c_{n}^{(2 n+\mu)}$ vanish (see (2.7a) and (2.7c)),

$$
c_{n}^{(2 n+\mu)}=b_{n-1}^{(2 n+\mu-2)}+g_{n}^{(2 n+\mu)} g_{n-1}^{(2 n+\mu-1)}=0,
$$

then the polynomials $p_{n}^{(2 n+\mu)}(x)$ factorize as follows:

$$
p_{n}^{(2 n+\mu)}(x)=\prod_{m=1}^{n}\left[x-x_{m}^{(2,2 m+\mu)}\right]
$$

entailing

$$
p_{0}^{(\mu)}(x)=1, \quad p_{1}^{(2+\mu)}(x)=x-x_{1}^{(2,2+\mu)}, \quad p_{2}^{(4+\mu)}(x)=\left[x-x_{1}^{(2,2+\mu)}\right]\left[x-x_{2}^{(2,4+\mu)}\right],
$$

and so on.

Likewise, if for all nonnegative integer values of $n$, the following two properties hold (see (2.8a), $(2.8 \mathrm{c})$, and $(2.8 \mathrm{~d}))$ :

$$
\begin{gathered}
d_{n}^{(3 n+\mu)}=b_{n-1}^{(3 n+\mu-3)}+g_{n}^{(3 n+\mu)} g_{n-1}^{(3 n+\mu-2)}+g_{n}^{(3 n+\mu-1)} g_{n-1}^{(3 n+\mu-2)}+g_{n}^{(3 n+\mu)} g_{n-1}^{(3 n+\mu-1)}=0 \\
e_{n}^{(3 n+\mu)}=0, \quad \text { that is, } g_{n}^{(3 n+\mu)}=0 \quad \text { or } \quad g_{n-1}^{(3 n+\mu-1)}=0 \quad \text { or } \quad g_{n-2}^{(3 n+\mu-2)}=0
\end{gathered}
$$


then the polynomials $p_{n}^{(3 n+\mu)}(x)$ factorize as follows:

$$
p_{n}^{(3 n+\mu)}(x)=\prod_{m=1}^{n}\left[x-x_{m}^{(3,3 m+\mu)}\right]
$$

entailing

$$
p_{0}^{(\mu)}(x)=1, \quad p_{1}^{(3+\mu)}(x)=x-x_{1}^{(3,3+\mu)}, \quad p_{2}^{(6+\mu)}(x)=\left[x-x_{1}^{(3,3+\mu)}\right]\left[x-x_{2}^{(3,6+\mu)}\right],
$$

and so on.

Here of course the $n$ (n-independent!) zeros $x_{m}^{(2,2 m+\mu)}$, respectively, $x_{m}^{(3,3 m+\mu)}$ are defined by $(2.7 \mathrm{~b})$, respectively, $(2.8 \mathrm{~b})$.

These findings correspond to [6, Proposition 2].

\section{Results for the Polynomials of the Askey Scheme}

In this section, we apply to the polynomials of the Askey scheme [9] the results reviewed in the previous section. This class of polynomials (including the classical polynomials) may be introduced in various manners: via generating functions, Rodriguez-type formulas, their connections with hypergeometric formulas, and so forth. In order to apply our machinery, as outlined in the preceding section, we introduce them via the three-term recursion relation they satisfy:

$$
p_{n+1}(x ; \underline{\eta})=\left[x+a_{n}(\underline{\eta})\right] p_{n}(x ; \underline{\eta})+b_{n}(\underline{\eta}) p_{n-1}(x ; \underline{\eta})
$$

with the "initial" assignments

$$
p_{-1}(x ; \underline{\eta})=0, \quad p_{0}(x ; \underline{\eta})=1,
$$

clearly entailing

$$
p_{1}(x ; \underline{\eta})=x+a_{0}(\underline{\eta}), \quad p_{2}(x ; \underline{\eta})=\left[x+a_{1}(\underline{\eta})\right]\left[x+a_{0}(\underline{\eta})\right]+b_{1}(\underline{\eta}),
$$

and so on. Here the components of the vector $\eta$ denote the additional parameters generally featured by these polynomials.

Let us emphasize that in this manner we introduced the monic (or "normalized" [9]) version of these polynomials; below we generally also report the relation of this version to the more standard version [9].

To apply our machinery we must identify, among the parameters characterizing these polynomials, the single parameter $v$ playing a special role in our approach. This can be generally done in several ways (even for the same class of polynomials, see below). Once this 
identification (i.e., the assignment $\eta \equiv \eta(v)$ ) has been made, the recursion relations (3.1) coincide with the relations (2.1) via the self-evident notational identification:

$$
p_{n}^{(v)}(x) \equiv p_{n}(x ; \underline{\eta}(v)), \quad a_{n}^{(v)} \equiv a_{n}(\underline{\eta}(v)), \quad b_{n}^{(v)} \equiv b_{n}(\underline{\eta}(v)) .
$$

Before proceeding with the report of our results, let us also emphasize that when the polynomials considered below feature symmetries regarding the dependence on their parameters-for instance, they are invariant under exchanges of some of them-obviously all the properties of these polynomials reported below can be duplicated via such symmetry properties; but it would be a waste of space to report explicitly the corresponding formulas, hence such duplications are hereafter omitted (except that sometimes results arrived at by different routes can be recognized as trivially related via such symmetries: when this happens this fact is explicitly noted). We will use systematically the notation of [9] — up to obvious changes made whenever necessary in order to avoid interferences with our previous notation. When we obtain a result that we deem interesting but is not reported in the standard compilations [9-13], we identify it as new (although given the very large literature on orthogonal polynomials, we cannot be certain that such a result has not been already published; indeed we will be grateful to any reader who were to discover that this is indeed the case and will let us know). And let us reiterate that even though we performed an extensive search for such results, this investigation cannot be considered "exhaustive": additional results might perhaps be discovered via assignments of the $v$-dependence $\underline{\eta}(v)$ different from those considered below.

\subsection{Wilson}

The monic Wilson polynomials (see [9], and note the notational replacement of the 4 parameters $a, b, c, d$ used there with $\alpha, \beta, \gamma, \delta)$

$$
p_{n}(x ; \alpha, \beta, \gamma, \delta) \equiv p_{n}(x ; \underline{\eta})
$$

are defined by the three-term recursion relations (3.1) with

$$
a_{n}(\underline{\eta})=\alpha^{2}-\tilde{A}_{n}-\tilde{C}_{n}, \quad b_{n}(\underline{\eta})=-\tilde{A}_{n-1} \tilde{C}_{n}
$$

where

$$
\begin{gathered}
\tilde{A}_{n}=\frac{(n+\alpha+\beta)(n+\alpha+\gamma)(n+\alpha+\delta)(n-1+\alpha+\sigma)}{(2 n-1+\alpha+\sigma)(2 n+\alpha+\sigma)}, \\
\tilde{C}_{n}=\frac{n(n-1+\beta+\gamma)(n-1+\beta+\delta)(n-1+\gamma+\delta)}{(2 n-2+\alpha+\sigma)(2 n-1+\alpha+\sigma)}, \\
\sigma \equiv \beta+\gamma+\delta, \quad \rho \equiv \beta \gamma+\beta \delta+\gamma \delta, \quad \tau \equiv \beta \gamma \delta .
\end{gathered}
$$


The standard version of these polynomials reads (see [9]):

$$
W_{n}(x ; \alpha, \beta, \gamma, \delta)=(-1)^{n}(n-1+\alpha+\beta+\gamma+\delta)_{n} p_{n}(x ; \alpha, \beta, \gamma, \delta) \text {. }
$$

Let us also recall that these polynomials $p_{n}(x ; \alpha, \beta, \gamma, \delta)$ are invariant under any permutation of the 4 parameters $\alpha, \beta, \gamma, \delta$.

As for the identification of the parameter $v$ (see (3.2)), two possibilities are listed in the following subsections.

\subsubsection{First Assignment}

$$
\alpha=-v .
$$

With this assignment, one can set, consistently with our previous treatment,

$$
\begin{aligned}
A_{n}^{(v)}=[6(2 n-2-v+\sigma)]^{-1} n\{ & 4-5 \sigma+6 \rho-6 \tau+(5-6 \sigma+6 \rho) \mathcal{v} \\
+ & {[-10+9 \sigma-6 \rho+(-9+6 \sigma) v] n } \\
+ & \left.(8-4 \sigma+4 v) n^{2}-2 n^{3}\right\} \\
\omega^{(v)} & =-v^{2}
\end{aligned}
$$

implying, via (2.2), (2.3), that the polynomials $p_{n}^{(v)}(x)$ defined by the three-term recurrence relations (2.1) coincide with the normalized Wilson polynomials (3.3):

$$
p_{n}^{(v)}(x)=p_{n}(x ;-v, \beta, \gamma, \delta)
$$

Hence, with this identification, Proposition 2.1 becomes applicable, entailing (new finding) that these normalized Wilson polynomials satisfy the second recursion relation (2.4a) with

$$
g_{n}^{(v)}=\frac{n(n-1+\beta+\gamma)(n-1+\beta+\delta)(n-1+\gamma+\delta)}{(2 n-2-v+\sigma)(2 n-1-v+\sigma)}
$$

Note that this finding is obtained without requiring any limitation on the 4 parameters of the Wilson polynomials $p_{n}(x ; \alpha, \beta, \gamma, \delta)$.

It is, moreover, plain that with the assignment

$$
v=n-1+\beta, \text { namely, } \alpha=-n+1-\beta,
$$

the factorizations implied by Proposition 2.3, and the properties implied by Corollary 2.4, become applicable with $\mu=\beta-1$. These are new findings. As for the additional findings 
entailed by Corollary 2.5, they are reported in Section 3.1.3. And Proposition 2.7 becomes as well applicable, entailing (new finding) the Diophantine factorization

$$
p_{n}(x ;-n+1-\beta, \beta, \gamma, \delta)=\prod_{m=1}^{n}\left[x+(m-1+\beta)^{2}\right],
$$

while Corollary 2.8 entails even more general properties, such as (new finding)

$$
p_{n}\left[-(\ell-1+\beta)^{2} ;-m+1-\beta, \beta, \gamma, \delta\right]=0, \quad \ell=1, \ldots, m, m=1, \ldots, n .
$$

Remark 3.1. A look at the formulas (3.3) suggests other possible assignments of the parameter $v$ satisfying (2.9), such as $v=n-2+\sigma$, namely, $\alpha=2-n-\sigma$. However, these assignments actually fail to satisfy (2.9) for all values of $n$, because for this to happen, it is not sufficient that the numerator in the expression of $b_{n}^{(v+\mu)}$ vanish, it is, moreover, required that the denominator in that expression never vanish. In the following, we will consider only assignments of the parameter $v$ in terms of $n$ that satisfy these requirements.

\subsubsection{Second Assignment}

$$
\alpha=-\frac{v}{2}, \quad \beta=\frac{1-v}{2} .
$$

With this assignment, one can set, consistently with our previous treatment,

$$
\begin{gathered}
A_{n}^{(v)}=[6(4 n-3-2 v+2 \gamma+2 \delta)]^{-1} n\left\{3-4 \gamma-4 \delta+6 \gamma \delta+(7-9 \gamma-9 \delta+12 \gamma \delta) v+3(1-\gamma-\delta) v^{2}\right. \\
-\left[11-12 \gamma-12 \delta+12 \gamma \delta+3(5-4 \gamma-4 \delta) v+3 v^{2}\right] n \\
\left.+4(3+2 v-2 \gamma-2 \delta) n^{2}-4 n^{3}\right\} \\
\omega^{(v)}=-\frac{v^{2}}{4}
\end{gathered}
$$

implying, via (2.2), (2.3), that the polynomials $p_{n}^{(v)}(x)$ defined by the three-term recurrence relations (2.1) coincide with the normalized Wilson polynomials (3.3):

$$
p_{n}^{(v)}(x)=p_{n}\left(x ;-\frac{v}{2}, \frac{1-v}{2}, \gamma, \delta\right)
$$

Hence, with this identification, Proposition 2.1 becomes applicable, entailing (new finding) that these normalized Wilson polynomials satisfy the second recursion relation (2.4a) with

$$
g_{n}^{(v)}=\frac{n(n-1+\gamma+\delta)(2 n-1-v+2 \gamma)(2 n-1-v+2 \delta)}{(4 n-3-2 v+2 \gamma+2 \delta)(4 n-1-2 v+2 \gamma+2 \delta)} \text {. }
$$


Note that this assignment entails now the (single) restriction $\beta=\alpha+1 / 2$ on the 4 parameters of the Wilson polynomials $p_{n}(x ; \alpha, \beta, \gamma, \delta)$.

It is, moreover, plain that with the assignments

$$
\begin{gathered}
v=n-\frac{1}{2}, \quad \text { hence } \alpha=-\frac{n}{2}+\frac{1}{4}, \quad \beta=-\frac{n}{2}+\frac{3}{4}, \\
v=n-2+2 \delta, \quad \gamma=\delta-\frac{1}{2}, \quad \alpha=-\frac{n}{2}+1-\delta, \quad \beta=-\frac{n}{2}+\frac{3}{2}-\delta,
\end{gathered}
$$

respectively,

$$
v=n-1+2 \delta, \quad \gamma=\delta+\frac{1}{2}, \quad \alpha=-\frac{n}{2}+\frac{1}{2}-\delta, \quad \beta=-\frac{n}{2}+1-\delta,
$$

the factorizations implied by Proposition 2.3 and the properties implied by Corollary 2.4 become applicable with $\mu=-1 / 2, \mu=-2+2 \delta$, respectively, $\mu=-1+2 \delta$. These are new findings. As for the additional findings entailed by Corollary 2.5, they are reported in Section 3.1.3. And Proposition 2.7 becomes as well applicable, entailing the Diophantine factorizations

$$
\begin{gathered}
p_{n}\left(x ;-\frac{n}{2}+\frac{1}{4},-\frac{n}{2}+\frac{3}{4}, \gamma, \delta\right)=\prod_{m=1}^{n}\left[x+\left(\frac{2 m-1}{4}\right)^{2}\right] \\
p_{n}\left(x ;-\frac{n}{2}+1-\delta,-\frac{n}{2}+\frac{3}{2}-\delta, \delta-\frac{1}{2}, \delta\right)=\prod_{m=1}^{n}\left[x+\left(\frac{m-2+2 \delta}{2}\right)^{2}\right],
\end{gathered}
$$

respectively,

$$
p_{n}\left(x ;-\frac{n}{2}+\frac{1}{2}-\delta,-\frac{n}{2}+1-\delta, \delta+\frac{1}{2}, \delta\right)=\prod_{m=1}^{n}\left[x+\left(\frac{m-1+2 \delta}{2}\right)^{2}\right] .
$$

(A referee pointed out that (3.17a) is not new, as one can evaluate explicitly $p_{n}(x ; \alpha, \beta, \gamma, \delta)$ when $n+\alpha+\beta+1=0$, which is indeed the case in (3.17a); and, moreover, that the two formulas $(3.17 \mathrm{~b})$ and (3.17c) coincide, since their left-hand sides are identical as a consequence of the symmetry property of Wilson polynomials under the transformation $\delta \Rightarrow \delta+1 / 2$.)

And Corollary 2.8 entails even more general properties, such as (new finding)

$$
\begin{gathered}
p_{n}\left[-\left(\frac{2 \ell-1}{4}\right)^{2} ;-\frac{m}{2}+\frac{1}{4},-\frac{m}{2}+\frac{3}{4}, \gamma, \delta\right]=0, \quad \ell=1, \ldots, m, m=1, \ldots, n, \\
p_{n}\left[-\left(\frac{\ell-2+2 \delta}{2}\right)^{2} ;-\frac{m}{2}+1-\delta,-\frac{m}{2}+\frac{3}{2}-\delta, \delta-\frac{1}{2}, \delta\right]=0, \quad \ell=1, \ldots, m, m=1, \ldots, n,
\end{gathered}
$$


respectively,

$$
p_{n}\left[-\left(\frac{\ell-1+2 \delta}{2}\right)^{2} ;-\frac{m}{2}+\frac{1}{2}-\delta,-\frac{m}{2}+1-\delta, \delta+\frac{1}{2}, \delta\right]=0, \quad \ell=1, \ldots, m, m=1, \ldots, n
$$

Moreover, with the assignments

$$
v=2 n-2+2 \delta, \quad \alpha=-n+1-\delta, \quad \beta=-n+\frac{3}{2}-\delta,
$$

respectively,

$$
v=2 n-1+2 \delta, \quad \alpha=-n+\frac{1}{2}-\delta, \quad \beta=-n+1-\delta,
$$

Proposition 2.9 becomes applicable, entailing (new findings) the Diophantine factorizations

$$
p_{n}\left(x ;-n+1-\delta,-n+\frac{3}{2}-\delta, \gamma, \delta\right)=\prod_{m=1}^{n}\left[x+(m-1+\delta)^{2}\right],
$$

respectively,

$$
p_{n}\left(x ;-n+\frac{1}{2}-\delta,-n+1-\delta, \gamma, \delta\right)=\prod_{m=1}^{n}\left[x+(m-1+\delta)^{2}\right]
$$

obviously implying the relation

$$
p_{n}\left(x ;-n+1-\delta,-n+\frac{3}{2}-\delta, \gamma, \delta\right)=p_{n}\left(x ;-n+\frac{1}{2}-\delta,-n+1-\delta, \gamma, \delta\right) .
$$

\subsubsection{Factorizations}

The following new relations among monic Wilson polynomials are implied by Proposition 2.3 with Corollary 2.5:

$$
\begin{aligned}
& p_{n}(x ;-m+1-\beta, \beta, \gamma, \delta) \\
& \quad=p_{n-m}(x ; m+\beta, \gamma, 1-\beta, \delta) p_{m}(x ;-m+1-\beta, \beta, \gamma, \delta), \quad m=0,1, \ldots, n, \\
& p_{n}\left(x ;-\frac{m}{2}+1-\delta,-\frac{m}{2}+\frac{3}{2}-\delta, \delta-\frac{1}{2}, \delta\right) \\
& \quad=p_{n-m}\left(x ; \frac{m}{2}-\frac{1}{2}+\delta, \frac{m}{2}+\delta, 1-\delta,-\delta+\frac{3}{2}\right) p_{m}\left(x ;-\frac{m}{2}+1-\delta,-\frac{m}{2}+\frac{3}{2}-\delta, \delta-\frac{1}{2}, \delta\right), \\
& m=0,1, \ldots, n .
\end{aligned}
$$


Note that the polynomials appearing as second factors in the right-hand side of these formulas are completely factorizable, see (3.10) and (3.17b) (we will not repeat this remark in the case of analogous formulas below).

\subsection{Racah}

The monic Racah polynomials (see [9])

$$
p_{n}(x ; \alpha, \beta, \gamma, \delta) \equiv p_{n}(x ; \underline{\eta})
$$

are defined by the three-term recursion relations (3.1) with

$$
a_{n}(\underline{\eta})=\tilde{A}_{n}+\widetilde{C}_{n}, \quad b_{n}(\underline{\eta})=-\tilde{A}_{n-1} \widetilde{C}_{n}
$$

where

$$
\begin{gathered}
\tilde{A}_{n}=\frac{(n+1+\alpha)(n+1+\alpha+\beta)(n+1+\beta+\delta)(n+1+\gamma)}{(2 n+1+\alpha+\beta)(2 n+2+\alpha+\beta)}, \\
\tilde{C}_{n}=\frac{n(n+\alpha+\beta-\gamma)(n+\alpha-\delta)(n+\beta)}{(2 n+\alpha+\beta)(2 n+1+\alpha+\beta)} .
\end{gathered}
$$

The standard version of these polynomials reads (see [9])

$$
R_{n}(x ; \alpha, \beta, \gamma, \delta)=\frac{(n+\alpha+\beta+1)_{n}}{(\alpha+1)_{n}(\beta+\delta+1)_{n}(\gamma+1)_{n}} p_{n}(x ; \alpha, \beta, \gamma, \delta) .
$$

Note, however, that in the following we do not require the parameters of these polynomials to satisfy one of the restrictions $\alpha=-N, \beta+\delta=-N$, or $\gamma=-N$, with $N$ a positive integer and $n=0,1, \ldots, N$, whose validity is instead required for the standard Racah polynomials [9].

Let us recall that these polynomials are invariant under various reshufflings of their parameters:

$$
\begin{aligned}
p_{n}(x ; \alpha, \beta, \gamma, \delta) & =p_{n}(x ; \alpha, \beta, \beta+\delta, \gamma-\beta) \\
& =p_{n}(x ; \beta+\delta, \alpha-\delta, \gamma, \delta) \\
& =p_{n}(x ; \gamma, \alpha+\beta-\gamma, \alpha,-\alpha+\gamma+\delta) .
\end{aligned}
$$

Let us now identify the parameter $v$ as follows (see (3.2)):

$$
\alpha=-v \text {. }
$$


With this assignment, one can set, consistently with our previous treatment,

$$
\begin{aligned}
& A_{n}^{(v)}=[6(2 n-v+\beta)]^{-1} n\{ \beta(2+3 \gamma+3 \delta)-[2+3(\gamma+\delta)+6 \gamma(\beta+\delta)] v \\
&+ {[4+6(\gamma+\delta)+3(\beta \gamma-\beta \delta+2 \gamma \delta)-3(2 \beta+\gamma+\delta) \mathcal{v}] n } \\
&+\left.4(-v+\beta) n^{2}+2 n^{3}\right\}, \\
& \omega^{(v)}=(v-1)(v+\gamma+\delta),
\end{aligned}
$$

implying, via (2.2), (2.3), that the polynomials $p_{n}^{(v)}(x)$ defined by the three-term recurrence relations (2.1) coincide with the normalized Racah polynomials (3.22):

$$
p_{n}^{(v)}(x)=p_{n}(x ;-v, \beta, \gamma, \delta)
$$

Hence, with this identification, Proposition 2.1 becomes applicable, entailing (new finding) that these normalized Racah polynomials satisfy the second recursion relation (2.4a) with

$$
g_{n}^{(v)}=-\frac{n(n+\beta)(n+\beta+\delta)(n+\gamma)}{(2 n-v+\beta)(2 n+1-v+\beta)}
$$

Note that this finding is obtained without requiring any limitation on the 4 parameters of the Racah polynomials $p_{n}(x ; \alpha, \beta, \gamma, \delta)$.

It is, moreover, plain that with the assignments

$$
\begin{aligned}
v=n, & \text { hence } \alpha=-n, \\
v=n-\delta, & \text { hence } \alpha=-n+\delta,
\end{aligned}
$$

respectively,

$$
v=n+\beta-\gamma, \text { hence } \alpha=-n-\beta+\gamma,
$$

the factorizations implied by Proposition 2.3 and the properties implied by Corollary 2.4 become applicable with $\mu=0, \mu=-\delta$, respectively, $\mu=\beta-\gamma$. These are new findings. As for the additional findings entailed by Corollary 2.5, they are reported in Section 3.2.1. And Proposition 2.7 becomes as well applicable, entailing (new findings) the Diophantine factorizations

$$
\begin{gathered}
p_{n}(x ;-n, \beta, \gamma, \delta)=\prod_{m=1}^{n}[x-(m-1)(m+\gamma+\delta)], \\
p_{n}(x ;-n+\delta, \beta, \gamma, \delta)=\prod_{m=1}^{n}[x-(m+\gamma)(m-\delta-1)],
\end{gathered}
$$


respectively,

$$
p_{n}(x ;-n-\beta+\gamma, \beta, \gamma, \delta)=\prod_{m=1}^{n}[x-(m-1+\beta-\gamma)(m+\beta+\delta)] .
$$

And Corollary 2.8 entails even more general properties, such as (new findings)

$$
\begin{gathered}
p_{n}[(\ell-1)(\ell+\gamma+\delta) ;-m, \beta, \gamma, \delta]=0, \quad \ell=1, \ldots, m, m=1, \ldots, n, \\
p_{n}[(\ell+\gamma)(\ell-\delta-1) ;-m+\delta, \beta, \gamma, \delta]=0, \quad \ell=1, \ldots, m, m=1, \ldots, n,
\end{gathered}
$$

respectively,

$$
p_{n}[(\ell-1+\beta-\gamma)(\ell+\beta+\delta) ;-m-\beta+\gamma, \beta, \gamma, \delta]=0, \quad \ell=1, \ldots, m, m=1, \ldots, n .
$$

\subsubsection{Factorizations}

The following new relations among Racah polynomials are implied by Proposition 2.3 with Corollary 2.5:

$$
\begin{aligned}
& p_{n}(x ;-m, \beta,-1,1)=p_{n-m}(x ; m, \beta,-1,1) p_{m}(x ;-m, \beta,-1,1), \quad m=0,1, \ldots, n, \\
& p_{n}(x ;-m+\delta, \beta,-\delta, \delta)=p_{n-m}(x ; m-\delta, 2 \delta+\beta, \delta,-\delta) p_{m}(x ;-m+\delta, \beta,-\delta, \delta), \quad m=0,1, \ldots, n, \\
& p_{n}(x ;-m-\beta+\gamma, \beta, \gamma, c-\gamma) \\
& \quad=p_{n-m}(x ; m+\beta-\gamma+c,-\beta+2 \gamma-c, \gamma, c-\gamma) p_{m}(x ; v-m-\beta+\gamma, \beta, \gamma, c-\gamma), \quad m=0,1, \ldots, n, \\
& \quad p_{n}(x ; \alpha,-m, \gamma, \delta)=p_{n-m}(x ; \alpha, m, \delta, \gamma) p_{m}(x ; \alpha,-m, \gamma, \delta), \quad m=0,1, \ldots, n, \\
& p_{n}(x, \alpha,-m-\alpha+\eta, \eta, \delta)=p_{n-m}(x, \eta, m, \eta+\delta-\alpha, \alpha) p_{m}(x, \alpha,-m-\alpha+\eta, \eta, \delta), \quad m=0,1, \ldots, n .31
\end{aligned}
$$

\subsection{Continuous Dual Hahn (CDH)}

In this section (some results of which were already reported in [5]) we focus on the monic continuous dual Hahn $(\mathrm{CDH})$ polynomials $p_{n}(x ; \alpha, \beta, \gamma)$ (see [9], and note the notational replacement of the 3 parameters $a, b, c$ used there with $\alpha, \beta, \gamma)$,

$$
p_{n}(x ; \alpha, \beta, \gamma) \equiv p_{n}(x ; \underline{\eta})
$$

defined by the three-term recursion relations (3.1) with

$$
\begin{aligned}
& a_{n}(\underline{\eta})=\alpha^{2}-(n+\alpha+\beta)(n+\alpha+\gamma)-n(n-1+\beta+\gamma), \\
& b_{n}(\underline{\eta})=-n(n-1+\alpha+\beta)(n-1+\alpha+\gamma)(n-1+\beta+\gamma) .
\end{aligned}
$$


The standard version of these polynomials reads (see [9])

$$
S_{n}(x ; \alpha, \beta, \gamma)=(-1)^{n} p_{n}(x ; \alpha, \beta, \gamma)
$$

Let us recall that these polynomials $p_{n}(x ; \alpha, \beta, \gamma)$ are invariant under any permutation of the three parameters $\alpha, \beta, \gamma$.

Let us now proceed and provide two identifications of the parameter $v$, see (3.2).

\subsubsection{First Assignment}

$$
\alpha=-v \text {. }
$$

With this assignment, one can set, consistently with our previous treatment,

$$
\begin{gathered}
A_{n}^{(v)}=n\left[-\frac{5}{6}+\beta+\gamma-\beta \gamma+(\beta+\gamma-1) v+\left(\frac{3}{2}-\beta-\gamma+v\right) n-\frac{2}{3} n^{2}\right], \\
\omega^{(v)}=-v^{2},
\end{gathered}
$$

implying, via (2.2), (2.3), that the polynomials $p_{n}^{(v)}(x)$ defined by the three-term recurrence relations (2.1) coincide with the normalized $\mathrm{CDH}$ polynomials (3.32):

$$
p_{n}^{(v)}(x)=p_{n}(x ;-v, \beta, \gamma)
$$

Hence, with this identification, Proposition 2.1 becomes applicable, entailing (new finding) that these normalized $\mathrm{CDH}$ polynomials satisfy the second recursion relation (2.4a) with

$$
g_{n}^{(v)}=n(n-1+\beta+\gamma)
$$

Note that this finding is obtained without requiring any limitation on the 3 parameters of the $\mathrm{CDH}$ polynomials $p_{n}(x ; \alpha, \beta, \gamma)$.

It is, moreover, plain that with the assignment

$$
v=n-1+\beta, \quad \text { hence } \alpha=-n+1-\beta,
$$

the factorizations implied by Proposition 2.3 and the properties implied by Corollary 2.4 become applicable with $\mu=-1+\beta$. These are new findings. As for the additional findings entailed by Corollary 2.5, they are reported in Section 3.3.3. And Proposition 2.7 becomes as well applicable, entailing (new findings) the Diophantine factorization

$$
p_{n}(x ;-n+1-\beta, \beta, \gamma)=\prod_{m=1}^{n}\left[x+(m-1+\beta)^{2}\right] .
$$


And Corollary 2.8 entails even more general properties, such as (new finding)

$$
p_{n}\left[-(\ell-1+\beta)^{2} ;-m+1-\beta, \beta, \gamma\right]=0, \quad \ell=1, \ldots, m, m=1, \ldots, n .
$$

Likewise, with the assignment

$$
v=2 n+\beta, \quad \alpha=-2 n-\beta, \quad \gamma=\frac{1}{2}
$$

Proposition 2.9 becomes applicable, entailing (new finding) the Diophantine factorization

$$
p_{n}\left(x ;-2 n-\beta, \beta, \frac{1}{2}\right)=\prod_{m=1}^{n}\left[x+(2 m-1+\beta)^{2}\right] .
$$

\subsubsection{Second Assignment}

$$
\alpha=-\frac{1}{2} v+c, \quad \beta=-\frac{1}{2}(v+1)+c
$$

where $c$ is an a priori arbitrary parameter.

With this assignment, one can set, consistently with our previous treatment,

$$
\begin{gathered}
A_{n}^{(v)}=n\left[-\frac{4}{3}+\frac{3}{2} \gamma+\frac{5}{2} c-c^{2}-2 \gamma c+\left(-\frac{5}{4}+\gamma+c\right) v-\frac{1}{4} v^{2}+(2-\gamma-2 c+v) n-\frac{2}{3} n^{2}\right] \\
\omega^{(v)}=-\frac{1}{4}(1-2 c+v)^{2}
\end{gathered}
$$

implying, via (2.2), (2.3), that the polynomials $p_{n}^{(v)}(x)$ defined by the three-term recurrence relations (2.1) coincide with the normalized $\mathrm{CDH}$ polynomials (3.32):

$$
p_{n}^{(v)}(x)=p_{n}\left(x ; c-\frac{v}{2}, c-\frac{v}{2}-\frac{1}{2}, \gamma\right)
$$

Hence, with this identification, Proposition 2.1 becomes applicable, entailing (new finding) that these normalized $\mathrm{CDH}$ polynomials satisfy the second recursion relation (2.4a) with

$$
g_{n}^{(v)}=n\left(n-1-\frac{v}{2}+\gamma+c\right)
$$

Note that this assignment entails the (single) limitation $\beta=\alpha-1 / 2$ on the parameters of the $\mathrm{CDH}$ polynomials. 
It is, moreover, plain that with the assignment

$$
v=n+2 c-\frac{3}{2}, \quad \text { hence } \alpha=-\frac{n}{2}+\frac{3}{4}, \quad \beta=-\frac{n}{2}+\frac{1}{4},
$$

the factorizations implied by Proposition 2.3 and the properties implied by Corollary 2.4 become applicable with $\mu=2 c-3 / 2$. These are new findings. As for the additional findings entailed by Corollary 2.5, they are reported in Section 3.3.3. And Proposition 2.7 becomes as well applicable, entailing (new findings) the Diophantine factorization

$$
p_{n}\left(x ;-\frac{n}{2}+\frac{3}{4},-\frac{n}{2}+\frac{1}{4}, \gamma\right)=\prod_{m=1}^{n}\left[x+\left(\frac{2 m-1}{4}\right)^{2}\right]
$$

And Corollary 2.8 entails even more general properties, such as (new finding)

$$
p_{n}\left[-\left(\frac{2 \ell-1}{4}\right)^{2} ;-\frac{m}{2}+\frac{3}{4},-\frac{m}{2}+\frac{1}{4}, r\right]=0, \quad \ell=1, \ldots, m, m=1, \ldots, n
$$

Likewise with the assignments

$$
v=2(n-1+c+\gamma), \quad \text { hence } \alpha=-n+1-\gamma, \quad \beta=-n+\frac{1}{2}-\gamma,
$$

respectively,

$$
v=2\left(n-\frac{3}{2}+c+\gamma\right), \quad \text { hence } \alpha=-n+\frac{3}{2}-\gamma, \quad \beta=-n+1-\gamma,
$$

Proposition 2.9 becomes applicable, entailing (new findings) the Diophantine factorizations

$$
p_{n}\left(x ;-n+1-\gamma,-n+\frac{1}{2}-\gamma, \gamma\right)=\prod_{m=1}^{n}\left[x+(m-1+\gamma)^{2}\right]
$$

respectively,

$$
p_{n}\left(x ;-n+\frac{3}{2}-\gamma,-n+1-\gamma, \gamma\right)=\prod_{m=1}^{n}\left[x+(m-1+\gamma)^{2}\right]
$$

Note that the right-hand sides of the last two formulas coincide; this implies (new finding) that the left-hand sides coincide as well. 


\subsubsection{Factorizations}

The following new relations among continuous dual Hahn polynomials are implied by Proposition 2.3 with Corollary 2.5:

$$
p_{n}(x ;-m+1-\beta, \beta, \gamma)=p_{n-m}(x ; m+\beta, 1-\beta, \gamma) p_{m}(x ;-m+1-\beta, \beta, \gamma), \quad m=0,1, \ldots, n .
$$

\subsection{Continuous Hahn (CH)}

The monic continuous Hahn $(\mathrm{CDH})$ polynomials $p_{n}(x ; \alpha, \beta, \gamma, \delta)$ (see [9], and note the notational replacement of the 4 parameters $a, b, c, d$ used there with $\alpha, \beta, \gamma, \delta$ ),

$$
p_{n}(x ; \alpha, \beta, \gamma, \delta) \equiv p_{n}(x ; \underline{\eta})
$$

are defined by the three-term recursion relations (3.1) with

$$
a_{n}(\underline{\eta})=-i\left(\alpha+\tilde{A}_{n}+\tilde{C}_{n}\right), \quad b_{n}(\underline{\eta})=\tilde{A}_{n-1} \tilde{C}_{n}
$$

where

$$
\begin{aligned}
& \tilde{A}_{n}=-\frac{(n-1+\alpha+\beta+\gamma+\delta)(n+\alpha+\gamma)(n+\alpha+\delta)}{(2 n-1+\alpha+\beta+\gamma+\delta)(2 n+\alpha+\beta+\gamma+\delta)} \\
& \tilde{C}_{n}=\frac{n(n-1+\beta+\gamma)(n-1+\beta+\delta)}{(2 n+\alpha+\beta+\gamma+\delta-1)(2 n+\alpha+\beta+\gamma+\delta-2)}
\end{aligned}
$$

The standard version of these polynomials reads (see [9])

$$
S_{n}(x ; \alpha, \beta, \gamma, \delta)=(-1)^{n} p_{n}(x ; \alpha, \beta, \gamma, \delta) .
$$

Let us recall that these polynomials are symmetrical under the exchange of the first two and last two parameters:

$$
p_{n}(x ; \alpha, \beta, \gamma, \delta)=p_{n}(x ; \beta, \alpha, \gamma, \delta)=p_{n}(x ; \alpha, \beta, \delta, \gamma)=p_{n}(x ; \beta, \alpha, \delta, \gamma)
$$

Let us now proceed and provide two identifications of the parameter $v$, see (3.2).

\subsubsection{First Assignment}

$$
\alpha=-v \text {. }
$$


With this assignment, one can set, consistently with our previous treatment,

$$
\begin{gathered}
A_{n}^{(v)}=i n \frac{-\beta+\gamma+\delta-2 \gamma \delta+(1-2 \beta) v+(\beta-\gamma-\delta-v) n}{2(2-\beta-\gamma-\delta+v-2 n)}, \\
\omega^{(v)}=-i v,
\end{gathered}
$$

implying, via (2.2), (2.3), that the polynomials $p_{n}^{(v)}(x)$ defined by the three-term recurrence relations (2.1) coincide with the normalized $\mathrm{CH}$ polynomials (3.53):

$$
p_{n}^{(v)}(x)=p_{n}(x ;-v, \beta, \gamma, \delta)
$$

Hence, with this identification, Proposition 2.1 becomes applicable, entailing (new finding) that these normalized $\mathrm{CH}$ polynomials satisfy the second recursion relation (2.4a) with

$$
g_{n}^{(v)}=\frac{\operatorname{in}(n-1+\beta+\gamma)(n-1+\beta+\delta)}{(2 n-2-v+\beta+\gamma+\delta)(2 n-1-v+\beta+\gamma+\delta)} .
$$

Note that this assignment entails no restriction on the 4 parameters of the $\mathrm{CH}$ polynomials $p_{n}(x ; \alpha, \beta, \gamma, \delta)$.

It is, moreover, plain that with the assignment

$$
v=n-1+\gamma, \text { hence } \alpha=-n+1-\gamma,
$$

the factorizations implied by Proposition 2.3 and the properties implied by Corollary 2.4 become applicable with $\mu=-1+\gamma$. These are new findings. And Proposition 2.7 becomes as well applicable, entailing (new findings) the Diophantine factorization

$$
p_{n}(x ;-n+1-\gamma, \beta, \gamma, \delta)=\prod_{m=1}^{n}[x+i(m-1+\gamma)] .
$$

And Corollary 2.8 entails even more general properties, such as (new findings)

$$
p_{n}[-i(\ell-1+\gamma) ;-m+1-\gamma, \beta, \gamma, \delta]=0, \quad \ell=1, \ldots, m, m=1, \ldots, n .
$$

\subsubsection{Second Assignment}

Analogous results also obtain from the assignment

$$
\gamma=-v
$$


With this assignment, one can set, consistently with our previous treatment,

$$
\begin{gathered}
A_{n}^{(v)}=-\frac{i n[n(\alpha+\beta-\delta+v)+(2 \delta-1) v+\alpha(2 \beta-1)-\beta+\delta]}{2(2 n-2+\alpha+\beta+\delta-v)}, \\
\omega^{(v)}=i v,
\end{gathered}
$$

implying, via (2.2), (2.3), that the polynomials $p_{n}^{(v)}(x)$ defined by the three-term recurrence relations (2.1) coincide with the normalized $\mathrm{CH}$ polynomials (3.53):

$$
p_{n}^{(v)}(x)=p_{n}(x ; \alpha, \beta,-v, \delta)
$$

Hence, with this identification, Proposition 2.1 becomes applicable, entailing (new finding) that these normalized $\mathrm{CH}$ polynomials satisfy the second recursion relation (2.4a) with

$$
g_{n}^{(v)}=\frac{i n(n-1+\alpha+\beta)(n-1+\beta+\delta)}{(2 n-2-v+\alpha+\beta+\delta)(2 n-1-v+\alpha+\beta+\delta)} .
$$

Note that this assignment entails no restriction on the 4 parameters of the $\mathrm{CH}$ polynomials $p_{n}(x ; \alpha, \beta, \gamma, \delta)$.

It is, moreover, plain that with the assignment

$$
v=n-1+\alpha, \text { hence } \gamma=-n+1-\alpha,
$$

the factorizations implied by Proposition 2.3, and the properties implied by Corollary 2.4, become applicable with $\mu=-1+\alpha$. These are new findings. And Proposition 2.7 becomes as well applicable, entailing (new finding) the Diophantine factorization

$$
p_{n}(x ; \alpha, \beta,-n+1-\alpha, \delta)=\prod_{m=1}^{n}[x-i(m-1+\alpha)] .
$$

And Corollary 2.8 entails even more general properties, such as (new finding)

$$
p_{n}[i(\ell-1+\alpha) ; \alpha, \beta,-m+1-\alpha, \delta]=0, \quad \ell=1, \ldots, m, m=1, \ldots, n .
$$

\subsection{Hahn}

In this subsection, we introduce a somewhat generalized version of the standard (monic) Hahn polynomials. These (generalized) monic Hahn polynomials $p_{n}(x ; \alpha, \beta, \gamma)$ (see [9], and note the replacement of the integer parameter $N$ with the arbitrary parameter $\gamma$ : hence the standard Hahn polynomials are only obtained for $\gamma=N$ with $N$ a positive integer and $n=$ $1,2, \ldots, N)$,

$$
p_{n}(x ; \alpha, \beta, \gamma) \equiv p_{n}(x ; \underline{\eta})
$$


are defined by the three-term recursion relations (3.1) with

$$
a_{n}(\underline{\eta})=-\left(\tilde{A}_{n}+\tilde{C}_{n}\right), \quad b_{n}(\underline{\eta})=-\tilde{A}_{n-1} \tilde{C}_{n}
$$

where

$$
\begin{gathered}
\tilde{A}_{n}=\frac{(n+1+\alpha)(n+1+\alpha+\beta)(-n+\gamma)}{(2 n+1+\alpha+\beta)(2 n+2+\alpha+\beta)}, \\
\tilde{C}_{n}=\frac{n(n+1+\alpha+\beta+\gamma)(n+\beta)}{(2 n+\alpha+\beta)(2 n+1+\alpha+\beta)} .
\end{gathered}
$$

The standard version of these polynomials reads (see [9])

$$
Q_{n}(x ; \alpha, \beta, \gamma)=\frac{(n+1+\alpha+\beta)_{n}}{(1+\alpha)_{n}(-\gamma)_{n}} p_{n}(x ; \alpha, \beta, \gamma)
$$

Let us now proceed and provide three identifications of the parameter $v$, see (3.2).

\subsubsection{First Assignment}

$$
\alpha=-v \text {. }
$$

With this assignment, one can set, consistently with our previous treatment,

$$
\begin{gathered}
A_{n}^{(v)}=\frac{n[\beta+(1+2 \gamma) v-(\beta+2 \gamma+v) n]}{2(2 n-v+\beta)}, \\
\omega^{(v)}=v-1
\end{gathered}
$$

implying, via (2.2), (2.3), that the polynomials $p_{n}^{(v)}(x)$ defined by the three-term recurrence relations (2.1) coincide with the normalized Hahn polynomials (3.69):

$$
p_{n}^{(v)}(x)=p_{n}(x ;-v, \beta, \gamma)
$$

Hence, with this identification, Proposition 2.1 becomes applicable, entailing (new finding) that these normalized Hahn polynomials satisfy the second recursion relation (2.4a) with

$$
g_{n}^{(v)}=-\frac{n(n+\beta)(n-1-\gamma)}{(2 n-v+\beta)(2 n+1-v+\beta)} .
$$

Note that this assignment entails no restriction on the 3 parameters of the Hahn polynomials $p_{n}(x ; \alpha, \beta, \gamma)$. 
It is, moreover, plain that with the assignments

$$
v=n
$$

respectively,

$$
v=n+1+\beta+\gamma,
$$

the factorizations implied by Proposition 2.3 and the properties implied by Corollary 2.4 become applicable with $\mu=1+\beta+\gamma$. These are new findings. And Proposition 2.7 becomes as well applicable, entailing (new findings) the Diophantine factorizations

$$
p_{n}(x ; n, \beta, \gamma)=\prod_{m=1}^{n}(x-m+1),
$$

respectively,

$$
p_{n}(x ; n+1+\beta+\gamma, \beta, \gamma)=\prod_{m=1}^{n}(x-m-\beta-\gamma)
$$

And Corollary 2.8 entails even more general properties, such as (new findings)

$$
p_{n}(\ell-1 ; m, \beta, \gamma)=0, \quad \ell=1, \ldots, m, \quad m=1, \ldots, n,
$$

respectively,

$$
p_{n}(\ell+\beta+\gamma ; m+1+\beta+\gamma, \beta, \gamma)=0, \quad \ell=1, \ldots, m, m=1, \ldots, n .
$$

\subsubsection{Second Assignment}

$$
\beta=-v+\gamma+c,
$$

where $c$ is an arbitrary parameter.

With this assignment, one can set, consistently with our previous treatment,

$$
\begin{gathered}
A_{n}^{(v)}=-n \frac{\alpha-\gamma-c+v+2 \alpha \gamma+(-\alpha+c-v+3 \gamma) n}{2(\alpha+\gamma+c-v+2 n)}, \\
\omega^{(v)}=1-v+2 \gamma+c,
\end{gathered}
$$


implying, via (2.2), (2.3), that the polynomials $p_{n}^{(v)}(x)$ defined by the three-term recurrence relations (2.1) coincide with the normalized Hahn polynomials (3.69):

$$
p_{n}^{(v)}(x)=p_{n}(x ; \alpha,-v+\gamma+c, \gamma)
$$

Hence, with this identification, Proposition 2.1 becomes applicable, entailing (new finding) that these normalized Hahn polynomials satisfy the second recursion relation (2.4a) with

$$
g_{n}^{(v)}=\frac{n(n+\alpha)(n-1-\gamma)}{(2 n-v+\alpha+\gamma+c)(2 n+1-v+\alpha+\gamma+c)} .
$$

Note that this assignment entails no restriction on the 3 parameters of the Hahn polynomials $p_{n}(x ; \alpha, \beta, \gamma)$.

It is, moreover, plain that with the assignments

$$
v=n+\gamma+c, \text { hence } \beta=-n,
$$

respectively,

$$
v=n+1+\alpha+2 \gamma+c, \quad \text { hence } \beta=-(n+1+\alpha+\gamma),
$$

the factorizations implied by Proposition 2.3 and the properties implied by Corollary 2.4 become applicable with $\mu=\gamma+c$, respectively, $\mu=1+\alpha+2 \gamma+c$. These are new findings. And Proposition 2.7 becomes as well applicable, entailing (new findings) the Diophantine factorizations

$$
p_{n}(x ; \alpha,-n, \gamma)=\prod_{m=1}^{n}(x+m-1-\gamma),
$$

respectively,

$$
p_{n}(x ; \alpha,-n-1-\alpha-\gamma, \gamma)=\prod_{m=1}^{n}(x+m+\alpha) \text {. }
$$

And Corollary 2.8 entails even more general properties, such as (new findings)

$$
p_{n}(-\ell+1+\gamma ; \alpha,-m, \gamma)=0, \quad \ell=1, \ldots, m, m=1, \ldots, n,
$$

respectively,

$$
p_{n}(-\ell-\alpha ; \alpha,-m-1-\alpha-\gamma, \gamma)=0, \quad \ell=1, \ldots, m, m=1, \ldots, n \text {. }
$$




\subsubsection{Third Assignment}

$$
\beta=-v+c, \quad \gamma=v,
$$

where $c$ is an arbitrary parameter.

With this assignment, one can set, consistently with our previous treatment,

$$
\begin{gathered}
A_{n}^{(v)}=-\frac{n[v+\alpha-c+2 \alpha v+(v-\alpha+c) n]}{2(2 n-v+\alpha+c)}, \\
\omega^{(v)}=v,
\end{gathered}
$$

implying, via (2.2), (2.3), that the polynomials $p_{n}^{(v)}(x)$ defined by the three-term recurrence relations (2.1) coincide with the normalized Hahn polynomials (3.69):

$$
p_{n}^{(v)}(x)=p_{n}(x ; \alpha,-v+c, v)
$$

Hence, with this identification, Proposition 2.1 becomes applicable, entailing (new finding) that these normalized Hahn polynomials satisfy the second recursion relation (2.4a) with

$$
g_{n}^{(v)}=-\frac{n(n+\alpha)(n+1+\alpha+c)}{(2 n-v+\alpha+c)(2 n+1-v+\alpha+c)} .
$$

Note that this assignment entails no restriction on the 4 parameters of the Hahn polynomials $p_{n}(x ; \alpha, \beta, \gamma)$.

It is, moreover, plain that with the assignment

$$
v=n+c, \quad \beta=-n, \quad \gamma=n+c,
$$

the factorizations implied by Proposition 2.3 and the properties implied by Corollary 2.4 become applicable with $\mu=c$. These are new findings. And Proposition 2.7 becomes as well applicable, entailing (new finding) the Diophantine factorization

$$
p_{n}(x ; \alpha,-n, n+c)=\prod_{m=1}^{n}(x-m-c) .
$$

And Corollary 2.8 entails even more general properties, such as (new finding)

$$
p_{n}(\ell+c ; \alpha,-m, m+c)=0, \quad \ell=1, \ldots, m, m=1, \ldots, n .
$$

\subsection{Dual Hahn}

In this subsection, we introduce a somewhat generalized version of the standard (monic) dual Hahn polynomials. These (generalized) monic dual Hahn polynomials $p_{n}(x ; \gamma, \delta, \eta)$ (see [9], 
and note the replacement of the integer parameter $N$ with the arbitrary parameter $\eta$ : hence the standard Hahn polynomials are only obtained for $\eta=N$ with $N$ a positive integer and $n=1,2, \ldots, N)$,

$$
p_{n}(x ; \gamma, \delta, \eta) \equiv p_{n}(x ; \underline{\eta})
$$

are defined by the three-term recursion relations (3.1) with

$$
a_{n}(\underline{\eta})=\tilde{A}_{n}+\tilde{C}_{n}, \quad b_{n}(\underline{\eta})=-\tilde{A}_{n-1} \tilde{C}_{n}
$$

where

$$
\widetilde{A}_{n}=(n+1+\gamma)(n-\eta), \quad \widetilde{C}_{n}=n(n-1-\delta-\eta) .
$$

The standard version of these polynomials reads (see [9])

$$
R_{n}(x ; \gamma, \delta, \eta)=\frac{1}{(1+\gamma)_{n}(-\eta)_{n}} p_{n}(x ; \gamma, \delta, \eta)
$$

Let us now proceed and provide two identifications of the parameter $v$.

\subsubsection{First Assignment}

$$
\eta=v
$$

With this assignment, one can set, consistently with our previous treatment,

$$
\begin{gathered}
A_{n}^{(v)}=n\left[\frac{1}{3}+\frac{-\gamma+\delta}{2}-\gamma v-\left(1+v+\frac{-\gamma+\delta}{2}\right) n+\frac{2}{3} n^{2}\right], \\
\omega^{(v)}=v(1+v+\gamma+\delta),
\end{gathered}
$$

implying, via (2.2), (2.3), that the polynomials $p_{n}^{(v)}(x)$ defined by the three-term recurrence relations (2.1) coincide with the normalized dual Hahn polynomials (3.92):

$$
p_{n}^{(v)}(x)=p_{n}(x ; \gamma, \delta, v)
$$

Hence, with this identification, Proposition 2.1 becomes applicable, entailing (new finding) that these normalized dual Hahn polynomials satisfy the second recursion relation (2.4a) with

$$
g_{n}^{(v)}=-n(n+\gamma)
$$


Note that this assignment entails no restriction on the 3 parameters of the dual Hahn polynomials $p_{n}(x ; \gamma, \delta, \eta)$.

It is, moreover, plain that with the assignments

$$
v=n-1, \text { hence } \eta=n-1,
$$

(which is, however, incompatible with the requirement characterizing the standard dual Hahn polynomials: $\eta=N$ with $N$ a positive integer and $n=1,2, \ldots, N)$, respectively,

$$
v=n-1-\delta, \text { hence } \eta=n-1-\delta,
$$

the factorizations implied by Proposition 2.3 and the properties implied by Corollary 2.4 become applicable with $\mu=-1$, respectively, $\mu=-1-\delta$. These are new findings. As for the additional findings entailed by Corollary 2.5, they are reported in Section 3.6.3. And Proposition 2.7 becomes as well applicable, entailing (new findings) the Diophantine factorizations

$$
p_{n}(x ; \gamma, \delta, n-1)=\prod_{m=1}^{n}[x-(m-1)(m+\gamma+\delta)]
$$

respectively,

$$
p_{n}(x ; \gamma, \delta, n-1-\delta)=\prod_{m=1}^{n}[x-(m+\gamma)(m-1-\delta)]
$$

And Corollary 2.8 entails even more general properties, such as (new findings)

$$
p_{n}[(\ell-1)(\ell+\gamma+\delta) ; \gamma, \delta, m-1]=0, \quad \ell=1, \ldots, m, m=1, \ldots, n,
$$

respectively,

$$
p_{n}[(\ell+\gamma)(\ell-1-\delta) ; \gamma, \delta, m-1-\delta]=0, \quad \ell=1, \ldots, m, m=1, \ldots, n .
$$

While for

$$
v=2 n, \text { hence } \eta=2 n, \text { and moreover } \delta=r,
$$

respectively,

$$
v=2 n-\delta, \text { hence } \eta=2 n-\delta, \text { and moreover } \delta=-\gamma,
$$

Proposition 2.9 becomes applicable, entailing (new findings) the Diophantine factorizations

$$
p_{n}(x ; \gamma, \gamma, 2 n)=\prod_{m=1}^{n}[x-2(2 m-1)(m+\gamma)]
$$


respectively,

$$
p_{n}(x ; \gamma,-\gamma, 2 n+\gamma)=\prod_{m=1}^{n}[x-(2 m-1+\gamma)(2 m+\gamma)]
$$

\subsubsection{Second Assignment}

$$
\gamma=-v, \quad \delta=v+c
$$

With this assignment, one can set, consistently with our previous treatment,

$$
\begin{gathered}
A_{n}^{(v)}=n\left[\frac{1}{3}+(1+\eta) v+\frac{1}{2} c-\left(1+v+\eta+\frac{1}{2} c\right) n+\frac{2}{3} n^{2}\right], \\
\omega^{(v)}=(v-1)(v+c),
\end{gathered}
$$

implying, via (2.2), (2.3), that the polynomials $p_{n}^{(v)}(x)$ defined by the three-term recurrence relations (2.1) coincide with the normalized Dual Hahn polynomials (3.92):

$$
p_{n}^{(v)}(x)=p_{n}(x ;-v, v+c, \eta)
$$

Hence, with this identification, Proposition 2.1 becomes applicable, entailing (new finding) that these normalized dual Hahn polynomials satisfy the second recursion relation (2.4a) with

$$
g_{n}^{(v)}=-n(n-1-\eta)
$$

Note that this assignment entails no restriction on the 3 parameters of the dual Hahn polynomials $p_{n}(x ; \gamma, \delta, \eta)$.

It is, moreover, plain that with the assignments

$$
v=n, \quad \text { hence } \gamma=-n, \quad \delta=n+c,
$$

respectively,

$$
v=n-1-\eta-c, \quad \text { hence } \gamma=-n+1+\eta+c, \quad \delta=n-1-\eta
$$

the factorizations implied by Proposition 2.3 and the properties implied by Corollary 2.4 become applicable with $\mu=0$, respectively, $\mu=-1-\eta-c$. These are new findings. As for the additional findings entailed by Corollary 2.5, they are reported in Section 3.6.3. 
And Proposition 2.7 becomes as well applicable, entailing (new findings) the Diophantine factorizations

$$
p_{n}(x ;-n, n+c, \eta)=\prod_{m=1}^{n}[x-(m-1)(m+c)]
$$

respectively,

$$
p_{n}(x ;-n+1+\eta+c, n-1-\eta, \eta)=\prod_{m=1}^{n}[x-(m-1-\eta)(m-2-\eta-c)] .
$$

And Corollary 2.8 entails even more general properties, such as (new findings)

$$
p_{n}[(\ell-1)(\ell+c) ;-m, m+c, \eta]=0, \quad \ell=1, \ldots, m, m=1, \ldots, n,
$$

respectively,

$$
p_{n}[(\ell-1-\eta)(\ell-2-\eta-c) ;-m+1+\eta+c,-m-1-\eta, \eta]=0, \quad \ell=1, \ldots, m, m=1, \ldots, n
$$

While for

$$
v=2 n-\eta, \quad \text { hence } \gamma=-2 n+\eta, \text { and moreover } c=0, \quad \text { hence } \delta=2 n-\eta,
$$

respectively,

$v=2 n+1$, hence $\gamma=-2 n-1$, and moreover $c=-2(\eta+1), \quad$ hence $\delta=2 n-1-2 \eta$,

Proposition 2.9 becomes applicable, entailing (new findings) the Diophantine factorizations

$$
p_{n}(x ;-2 n+\eta, 2 n-\eta, \eta)=\prod_{m=1}^{n}[x-(2 m-2-\eta)(2 m-1-\eta)]
$$

respectively,

$$
p_{n}(x ;-2 n-1,2 n-1-2 \eta, \eta)=\prod_{m=1}^{n}[x-2(2 m-1)(m-1-\eta)] .
$$




\subsubsection{Factorizations}

The following new relations among dual Hahn polynomials are implied by Proposition 2.3 with Corollary 2.5:

$$
\begin{aligned}
& p_{n}(x ; \gamma,-\gamma, m-1)=p_{n-m}(x ; \gamma,-\gamma,-m-1) p_{m}(x ; \gamma,-\gamma, m-1), \quad m=0,1, \ldots, n, \\
& p_{n}(x ; \gamma, \delta, m-1-\delta)=p_{n-m}(x ; \delta, \gamma,-m-1-\gamma) p_{m}(x ; \gamma, \delta, m-1-\delta), \quad m=0,1, \ldots, n, \\
& \quad p_{n}(x ;-m, m, \eta)=p_{n-m}(x ; m,-m, \eta) p_{m}(x ;-m, m, \eta), \quad m=0,1, \ldots, n, \\
& p_{n}(x ;-m+1+\eta+c, m-1-\eta, \eta) \\
& \quad p_{n-m}(x ; m-1-\eta,-m+1+\eta+c,-\eta-c-2) p_{m}(x ;-m+1+\eta+c, m-1-\eta, \eta), \quad m=0,1, \ldots, n .
\end{aligned}
$$

\subsection{Shifted Meixner-Pollaczek (sMP)}

In this subsection, we introduce and treat a modified version of the standard (monic) Meixner-Pollaczek polynomials. The standard (monic) Meixner-Pollaczek (MP) polynomials $p_{n}(x ; \alpha, \lambda)($ see $[9])$,

$$
p_{n}(x ; \alpha, \lambda) \equiv p_{n}(x ; \underline{\eta})
$$

are defined by the three-term recursion relations (3.1) with

$$
\begin{gathered}
a_{n}(\underline{\eta})=\alpha(n+\lambda)=\frac{n+\lambda}{\tan \phi} \\
b_{n}(\underline{\eta})=-\frac{1}{4}\left(1+\alpha^{2}\right) n(n-1+2 \lambda)=-\frac{n(n-1+2 \lambda)}{4 \sin ^{2} \phi} .
\end{gathered}
$$

The standard version of these polynomials reads (see [9])

$$
P_{n}^{(\lambda)}(x ; \tan \phi)=\frac{(2 \sin \phi)^{n}}{n !} p_{n}(x ; \alpha, \lambda), \quad \alpha \equiv \frac{1}{\tan \phi} .
$$

However, we have not found any assignment of the parameters $\alpha$ and $\lambda$ in terms of $v$ allowing the application of our machinery. We, therefore, consider the (monic) "shifted Meixner-Pollaczek" (sMP) polynomials

$$
p_{n}(x ; \alpha, \lambda, \beta)=p_{n}(x+\beta ; \alpha, \lambda),
$$


defined of course via the three-term recursion relation (3.1) with

$$
\begin{gathered}
a_{n}(\underline{\eta})=\alpha(n+\lambda)+\beta=\frac{n+\lambda}{\tan \phi}+\beta, \\
b_{n}(\underline{\eta})=-\frac{1}{4}\left(1+\alpha^{2}\right) n(n-1+2 \lambda)=-\frac{n(n-1+2 \lambda)}{4 \sin ^{2} \phi} .
\end{gathered}
$$

Then, with the assignment

$$
\lambda=-\frac{1}{2}(v+c), \quad \beta=-\frac{1}{2} i(v+C)
$$

(entailing no restriction on the parameters $\alpha, \lambda, \beta$, in as much as the two parameters $c$ and $C$ are arbitrary), one can set, consistently with our previous treatment,

$$
A_{n}^{(v)}=\frac{1}{2} n(\alpha n-\alpha v-i v-i C-\alpha c-\alpha), \quad \omega^{(v)}=\frac{1}{2} i(2 v+c+C),
$$

implying, via (2.2), (2.3), that the polynomials $p_{n}^{(v)}(x)$ defined by the three-term recurrence relations (2.1) coincide with the normalized shifted Meixner-Pollaczek polynomials:

$$
p_{n}^{(v)}(x)=p_{n}\left(x ; \alpha,-\frac{1}{2}(v+c),-\frac{1}{2} i(v+C)\right) .
$$

Hence, with this identification, Proposition 2.1 becomes applicable, entailing (new finding) that these (normalized) shifted Meixner-Pollaczek polynomials satisfy the second recursion relation $(2.4 a)$ with

$$
g_{n}^{(v)}=-\frac{1}{2} n(\alpha+i)
$$

It is, moreover, plain that with the assignment

$$
v=n-1-c \text { hence, } \lambda=-\frac{1}{2}(n-1), \quad \beta=-\frac{1}{2} i(n-1-c+C),
$$

the factorizations implied by Proposition 2.3, and the properties implied by Corollary 2.4, become applicable with $\mu=-1-c$. And Proposition 2.7 becomes as well applicable, entailing (new finding) the Diophantine factorization

$$
p_{n}\left(x ; \alpha,-\frac{1}{2}(n-1),-\frac{1}{2} i(n-1-c+C)\right)=\prod_{m=1}^{n}\left[x-i\left(m-1+\frac{C-c}{2}\right)\right] .
$$


And Corollary 2.8 entails even more general properties, such as (new finding)

$$
p_{n}\left(i\left(l-1+\frac{C-c}{2}\right) ; \alpha,-\frac{1}{2}(m-1),-\frac{1}{2} i(m-1-c+C)\right)=0, \quad \ell=1, \ldots, m, m=1, \ldots, n .
$$

\subsection{Meixner}

In this section (some results of which were already reported in [5]), we focus on the monic Meixner polynomials $p_{n}(x ; \beta, c)$ (see [9]),

$$
p_{n}(x ; \beta, c) \equiv p_{n}(x ; \underline{\eta})
$$

defined by the three-term recursion relations (3.1) with

$$
a_{n}(\underline{\eta})=\frac{\beta c+(1+c) n}{c-1}, \quad b_{n}(\underline{\eta})=-\frac{c n(n-1+\beta)}{(c-1)^{2}}
$$

The standard version of these polynomials reads (see [9]):

$$
M_{n}(x ; \beta, c)=\frac{1}{(\beta)_{n}}\left(\frac{c-1}{c}\right)^{n} p_{n}(x ; \beta, c) .
$$

We now identify the parameter $v$ via the assignment

$$
\beta=-v
$$

One can then set, consistently with our previous treatment,

$$
A_{n}^{(v)}=\frac{n[1+c+2 c v-(1+c) n]}{2(1-c)}, \quad \omega^{(v)}=v
$$

implying, via (2.2), (2.3), that the polynomials $p_{n}^{(v)}(x)$ defined by the three-term recurrence relations (2.1) coincide with the normalized Meixner polynomials (3.25):

$$
p_{n}^{(v)}(x)=p_{n}(x ;-v, c)
$$

Hence, with this identification, Proposition 2.1 becomes applicable, entailing (new finding) that these normalized Meixner polynomials satisfy the second recursion relation (2.4a) with

$$
g_{n}^{(v)}=\frac{c n}{1-c} .
$$


Note that this assignment entails no restriction on the 2 parameters of the Meixner polynomials $p_{n}(x ; \beta, c)$.

It is, moreover, plain that with the assignment

$$
v=n-1, \text { hence } \beta=1-n,
$$

the factorizations implied by Proposition 2.3, and the properties implied by Corollary 2.4, become applicable with $\mu=-1$. These are new findings. And Proposition 2.7 becomes as well applicable, entailing (new finding) the Diophantine factorization

$$
p_{n}(x ; 1-n, c)=\prod_{m=1}^{n}(x-m+1)
$$

And Corollary 2.8 entails even more general properties, such as (new finding)

$$
p_{n}(\ell-1 ; 1-m, c)=0, \quad \ell=1, \ldots, m, m=1, \ldots, n .
$$

Likewise for

$$
v=2 n \text { hence } \beta=-2 n \text { and moreover } c=-1 \text {, }
$$

Proposition 2.9 becomes applicable, entailing (new finding) the Diophantine factorization

$$
p_{n}(x ;-2 n,-1)=\prod_{m=1}^{n}(x-2 m+1) .
$$

\subsection{Krawtchouk}

The monic Krawtchouk polynomials $p_{n}(x ; \alpha, \beta)$ (see [9]: and note the notational replacement of the parameters $p$ and $N$ used there with the parameters $\alpha$ and $\beta$ used here, implying that only when $\beta=N$ and $n=1,2, \ldots, N$ with $N$ a positive integer these polynomials $p_{n}(x ; \alpha, \beta)$ coincide with the standard Krawtchouk polynomials),

$$
p_{n}(x ; \alpha, \beta) \equiv p_{n}(x ; \underline{\eta})
$$

are defined by the three-term recursion relations (3.1) with

$$
a_{n}(\underline{\eta})=-\alpha \beta+n(2 \alpha-1), \quad b_{n}(\underline{\eta})=\alpha(1-\alpha) n(n-1-\beta) .
$$

The standard version of these polynomials reads (see [9])

$$
K_{n}(x ; \alpha, \beta)=\frac{1}{\alpha^{n}(-\beta)_{n}} p_{n}(x ; \alpha, \beta) .
$$


We now identify the parameter $v$ via the assignment

$$
\beta=v
$$

One can then set, consistently with our previous treatment,

$$
A_{n}^{(v)}=n\left[\frac{1}{2}-\alpha-\alpha v+\left(-\frac{1}{2}+\alpha\right) n\right], \quad \omega^{(v)}=v,
$$

implying, via (2.2), (2.3), that the polynomials $p_{n}^{(v)}(x)$ defined by the three-term recurrence relations (2.1) coincide with the normalized Krawtchouk polynomials (3.135):

$$
p_{n}^{(v)}(x)=p_{n}(x ; \alpha, v)
$$

Hence, with this identification, Proposition 2.1 becomes applicable, entailing (new finding) that these normalized Krawtchouk polynomials satisfy the second recursion relation (2.4a) with

$$
g_{n}^{(v)}=-\alpha n
$$

Note that this assignment entails no restriction on the 2 parameters of the Krawtchouk polynomials $p_{n}(x ; \alpha, \beta)$.

It is, moreover, plain that with the assignment

$$
v=n-1, \text { hence } \beta=n-1
$$

(which is, however, incompatible with the definition of the standard Krawtchouk polynomials: $\beta=N$ and $n=1,2, \ldots, N$ with $N$ a positive integer), the factorizations implied by Proposition 2.3, and the properties implied by Corollary 2.4, become applicable with $\mu=-1$. These are new findings. And Proposition 2.7 becomes as well applicable, entailing (new finding) the Diophantine factorization

$$
p_{n}(x ; \alpha, n-1)=\prod_{m=1}^{n}(x-m+1) .
$$

And Corollary 2.8 entails even more general properties, such as (new findings)

$$
p_{n}(\ell-1 ; \alpha, m-1)=0, \quad \ell=1, \ldots, m, \quad m=1, \ldots, n .
$$

Likewise for

$$
v=2 n \text { hence } \beta=2 n \text { and moreover } \alpha=\frac{1}{2}
$$


(which is also incompatible with the definition of the standard Krawtchouk polynomials: $\beta=N$ and $n=1,2, \ldots, N$ with $N$ a positive integer), Proposition 2.9 becomes applicable, entailing (new finding) the Diophantine factorization

$$
p_{n}(x ;-2 n,-1)=\prod_{m=1}^{n}(x-2 m+1) .
$$

\subsection{Jacobi}

In this section (most results of which were already reported in [5]), we focus on the monic Jacobi polynomials $p_{n}(x ; \alpha, \beta)$ (see [9]),

$$
p_{n}(x ; \alpha, \beta) \equiv p_{n}(x ; \underline{\eta})
$$

defined by the three-term recursion relations (3.1) with

$$
\begin{gathered}
a_{n}(\underline{\eta})=\frac{(\alpha+\beta)(\alpha-\beta)}{(2 n+\alpha+\beta)(2 n+\alpha+\beta+2)}, \\
b_{n}(\underline{\eta})=-\frac{4 n(n+\alpha)(n+\beta)(n+\alpha+\beta)}{(2 n+\alpha+\beta-1)(2 n+\alpha+\beta+1)(2 n+\alpha+\beta)^{2}} .
\end{gathered}
$$

The standard version of these polynomials reads (see [9])

$$
P_{n}^{(\alpha, \beta)}(x)=\frac{(n+\alpha+\beta+1)_{n}}{2^{n} n !} p_{n}(x ; \alpha, \beta) .
$$

Let us recall that for the Jacobi polynomials there holds the symmetry relation

$$
p_{n}(-x ; \beta, \alpha)=p_{n}(x ; \alpha, \beta)
$$

We now identify the parameter $v$ as follows:

$$
\alpha=-v
$$

With this assignment one can set, consistently with our previous treatment,

$$
A_{n}^{(v)}=-\frac{n(v+\beta)}{2 n-v+\beta^{\prime}}, \quad \omega^{(v)}=1,
$$

implying, via (2.2), (2.3), that the polynomials $p_{n}^{(v)}(x)$ defined by the three-term recurrence relations (2.1) coincide with the normalized Jacobi polynomials (3.146):

$$
p_{n}^{(v)}(x)=p_{n}(x ;-v, \beta)
$$


Hence, with this identification, Proposition 2.1 becomes applicable, entailing (well-known result) that these normalized Jacobi polynomials satisfy the second recursion relation (2.4a) with

$$
g_{n}^{(v)}=-\frac{2 n(n+\beta)}{(2 n-v+\beta)(2 n-v+\beta+1)} .
$$

It is, moreover, plain that with the assignment

$$
v=n, \text { hence } \alpha=-n,
$$

the factorizations implied by Proposition 2.3, and the properties implied by Corollary 2.4, become applicable with $\mu=0$. These seem new findings. As for the additional findings entailed by Corollary 2.5, they are reported in Section 3.10.1. And Proposition 2.7 becomes as well applicable, entailing (well-known result) the Diophantine factorization

$$
p_{n}(x ;-n, \beta)=(x-1)^{n} .
$$

And Corollary 2.8 entails even more general properties, such as the fact that the $m$ Jacobi polynomials $p_{n}(x ;-m, \beta), m=1, \ldots, n$, feature $x=1$ as a zero of order $m$.

\subsubsection{Factorizations}

The following (not new) relations among Jacobi polynomials are implied by Proposition 2.3 with Corollary 2.5 (and see (3.153), of which the following formula is a generalization, just as (1.1c) is a generalization of (1.1a)):

$$
p_{n}(x ;-m, \beta)=p_{n-m}(x ; m, \beta) p_{m}(x ;-m, \beta)=(x-1)^{m} p_{n-m}(x ; m, \beta), \quad m=0,1, \ldots, n .
$$

\subsection{Laguerre}

In this section (most results of which were already reported in [5]), we focus on the monic Laguerre polynomials $p_{n}(x ; \alpha)$ (see [9]),

$$
p_{n}(x ; \alpha) \equiv p_{n}(x ; \underline{\eta})
$$

defined by the three-term recursion relations (3.1) with

$$
a_{n}(\underline{\eta})=-(2 n+1+\alpha), \quad b_{n}(\underline{\eta})=-n(n+\alpha) .
$$

The standard version of these polynomials reads (see [9])

$$
L_{n}^{(\alpha)}(x)=\frac{(-1)^{n}}{n !} p_{n}(x ; \alpha)
$$


We now identify the parameter $v$ as follows:

$$
\alpha=-v \text {. }
$$

With this assignment, one can set, consistently with our previous treatment,

$$
A_{n}^{(v)}=-n(n-v), \quad \omega^{(v)}=0,
$$

implying, via (2.2), (2.3), that the polynomials $p_{n}^{(v)}(x)$ defined by the three-term recurrence relations (2.1) coincide with the normalized Laguerre polynomials (3.155):

$$
p_{n}^{(v)}(x)=p_{n}(x ;-v)
$$

Hence, with this identification, Proposition 2.1 becomes applicable, entailing (well-known result) that the normalized Laguerre polynomials satisfy the second recursion relation (2.4a) with

$$
g_{n}^{(v)}=n
$$

It is, moreover, plain that with the assignment

$$
v=n \text {, hence } \alpha=-n,
$$

the factorizations implied by Proposition 2.3, and the properties implied by Corollary 2.4, become applicable with $\mu=0$. These seem new findings. As for the additional findings entailed by Corollary 2.5, they are reported in Section 3.11.1. And Proposition 2.7 becomes as well applicable, entailing (well-known result) the formula (see (1.1a))

$$
p_{n}(x ;-n)=x^{n} .
$$

And Corollary 2.8 entails even more general properties, such as the fact that the $m$ Laguerre polynomials $p_{n}(x ;-m), m=1, \ldots, n$, feature $x=0$ as a zero of order $m$, see (1.1c) or, equivalently, the next formula.

\subsubsection{Factorizations}

The following (not new) relations among Laguerre polynomials are implied by Proposition 2.3 with Corollary 2.5 (and see (3.162), of which the following formula-already reported above, see (1.1c)—is a generalization):

$$
p_{n}(x ;-m)=p_{n-m}(x ; m) p_{m}(x ;-m)=x^{m} p_{n-m}(x ; m), \quad m=0,1, \ldots, n .
$$




\subsection{Modified Charlier}

In this subsection, we introduce and treat a modified version of the standard (monic) Charlier polynomials. The standard (monic) Charlier polynomials $p_{n}(x ; \alpha)$ (see [9]),

$$
p_{n}(x ; \alpha, \lambda) \equiv p_{n}(x ; \underline{\eta}),
$$

are defined by the three-term recursion relations (3.1) with

$$
a_{n}(\underline{\eta})=-n-\alpha, \quad b_{n}(\underline{\eta})=-n \alpha .
$$

The standard version of these polynomials reads (see [9])

$$
C_{n}(x ; \alpha)=(-\alpha)^{-n} p_{n}(x ; \alpha) .
$$

However, we have not found any assignment of the parameters $\alpha$ in terms of $v$ allowing the application of our machinery. To nevertheless proceed, we introduce the class of (monic) "modified Charlier" polynomials $p_{n}(x ; \alpha, \beta, \gamma)$ characterized by the three-term recursion relation (3.1) with

$$
a_{n}(\underline{\eta})=-\gamma(n+\alpha)+\beta, \quad b_{n}(\underline{\eta})=-\gamma^{2} n \alpha,
$$

that obviously reduce to the monic Charlier polynomials for $\beta=0, \gamma=1$. Assigning instead

$$
\beta=-v, \quad \gamma=-1,
$$

one can set, consistently with our previous treatment,

$$
A_{n}^{(v)}=\frac{1}{2} n(n-1-2 v+2 \alpha), \quad \omega^{(v)}=v,
$$

implying, via (2.2), (2.3), that the polynomials $p_{n}^{(v)}(x)$ defined by the three-term recurrence relations (2.1) coincide with these (monic) modified Charlier polynomials:

$$
p_{n}^{(v)}(x)=p_{n}(x ; \alpha,-v,-1) .
$$

Hence, with this identification, Proposition 2.1 becomes applicable, entailing (new finding) that these (monic) modified Charlier polynomials satisfy the second recursion relation (2.4a) with

$$
g_{n}^{(v)}=-n
$$

There does not seem to be any interesting results for the zeros of these polynomials. 


\section{Outlook}

Other classes of orthogonal polynomials to which our machinery is applicable, partly overlapping with those reported in this paper, have been identified by finding explicit classes of coefficients $a_{n}^{(v)}$ and $b_{n}^{(\mathcal{v})}$ (defining these classes of orthogonal polynomials via the threeterm recursion relations (2.1)) that do satisfy the nonlinear relations entailing the validity of the various propositions reported above. Hence, for these classes of orthogonal polynomials analogous results to those reported above hold, namely an additional three-term recursion relation involving shifts in the parameter $v$, and possibly as well factorizations identifying Diophantine zeros. These findings will be reported, hopefully soon, in a subsequent paper, where we also elucidate and exploit the connection about the machinery reported above and the wealth of known results on discrete integrable systems [18]. Other developments connected with the machinery reported above are as well under investigation, including inter alia other types of additional recursion relations satisfied by the classes of orthogonal polynomials defined by the three-term recursion relations (2.1) (for appropriate choices of the coefficients $a_{n}^{(v)}$ and $b_{n}^{(v)}$ ) and the investigation of other properties of such polynomialspossibly including the identification of ODEs satisfied by them.

\section{Appendix}

\section{A. A proof}

In this Appendix, for completeness, we provide a proof of the factorization (2.11) with (2.12) (see Proposition 2.3) although this proof is actually quite analogous to that provided (for the special case with $\mu=0$ ) in [5, Section 4]. We proceed by induction, assuming that (2.11) holds up to $n$, and then showing that it holds for $n+1$. Indeed, by using it in the right-hand side of the relation (2.1a) with $v=m$, we get

$$
p_{n+1}^{(m+\mu)}(x)=\left[\left(x+a_{n}^{(m+\mu)}\right) \tilde{p}_{n-m}^{(-m)}(x)+b_{n}^{(m+\mu)} \tilde{p}_{n-1-m}^{(-m)}(x)\right] p_{m}^{(m+\mu)}(x), \quad m=0,1, \ldots, n-1,
$$

and clearly by using the recursion relation (2.12a) the square bracket in the right-hand side of this equation can be replaced by $\tilde{p}_{n+1-m}^{(-m)}(x)$, yielding

$$
p_{n+1}^{(m+\mu)}(x)=\tilde{p}_{n+1-m}^{(-m)}(x) p_{m}^{(m+\mu)}(x), \quad m=0,1, \ldots, n+1 .
$$

Note that for $m=n+1$, this formula is an identity, since $\tilde{p}_{0}^{(-m)}(x)=1$, see (2.12b); likewise, this formula clearly also holds for $m=n$, provided that (2.9) holds, see (2.1a) with $m=n$ and (2.12c).

But this is just the formula (2.11) with $n$ replaced by $n+1$. Q. E. D.

Remark A.1. The hypothesis (2.9) has been used above, in this proof of Proposition 2.3, only to prove the validity of the final formula, (A.2), for $m=n$. Hence one might wonder whether this hypothesis, (2.9), was redundant, since the validity of the final formula (A.2) for $m=n$ seems to be implied by (A.1) with (2.12c) and (2.12b), without the need to invoke (2.9). But in fact, by setting $m=n$ in the basic recurrence relation (2.1a), it is clear that (2.12c) and (2.12b) only hold provided (2.9) also holds. 


\section{Acknowledgments}

It is a pleasure to acknowledge useful discussions with H. W. Capel, Frank Nijhoff, Peter van der Kampf and, last but not least, Paul Edward Spicer who provided us with a copy of his Ph.D. thesis entitled "On Orthogonal Polynomials and Related Discrete Integrable Systems." These interactions occurred mainly in July 2007 during the XIV Workshop on Nonlinear Evolution Equations and Dynamical Systems (NEEDS 2007), the organizers of which-David Gómez Ullate, Andy Hone, Sara Lombardo, and Joachim Puig-we also like to thank for the excellent organization and the pleasant working atmosphere of that meeting.

\section{References}

[1] F. Calogero, L. Di Cerbo, and R. Droghei, "On isochronous Bruschi-Ragnisco-Ruijsenaars-Toda lattices: equilibrium configurations, behaviour in their neighbourhood, Diophantine relations and conjectures," Journal of Physics A: Mathematical and General, vol. 39, no. 2, pp. 313-325, 2006.

[2] F. Calogero, L. Di Cerbo, and R. Droghei, "On isochronous Shabat-Yamilov-Toda lattices: equilibrium configurations, behavior in their neighborhood, Diophantine relations and conjectures," Physics Letters A, vol. 355, no. 4-5, pp. 262-270, 2006.

[3] F. Calogero, Isochronous Systems, Oxford University Press, Oxford, UK, 2008.

[4] M. Bruschi, F. Calogero, and R. Droghei, "Proof of certain Diophantine conjectures and identification of remarkable classes of orthogonal polynomials," Journal of Physics A: Mathematical and Theoretical, vol. 40, no. 14, pp. 3815-3829, 2007.

[5] M. Bruschi, F. Calogero, and R. Droghei, "Tridiagonal matrices, orthogonal polynomials and Diophantine relations: I," Journal of Physics A: Mathematical and Theoretical, vol. 40, no. 32, pp. $9793-$ 9817, 2007.

[6] M. Bruschi, F. Calogero, and R. Droghei, "Tridiagonal matrices, orthogonal polynomials and Diophantine relations: II," Journal of Physics A: Mathematical and Theoretical, vol. 40, no. 49, pp. 1475914772, 2007.

[7] J. Favard, "Sur les polynômes de Tchebicheff," Comptes Rendus de l'Académie des Sciences, vol. 200, pp. 2052-2053, 1935.

[8] M. E. H. Ismail, Classical and Quantum Orthogonal Polynomials in One Variable, vol. 98 of Encyclopedia of Mathematics and Its Applications, Cambridge University Press, Cambridge, UK, 2005.

[9] R. Koekoek and R. F. Swarttouw, "The Askey-scheme of hypergeometric orthogonal polynomials and its q-analogue," Tech. Rep. 94-05, Delft University of Technology, Delft, The Netherlands, 1994, http:/ /aw.twi.tudelft.nl/ koekoek/askey.html.

[10] A. Erdélyi, Ed., Higher Transcendental Functions, McGraw-Hill, New York, NY, USA, 1953.

[11] I. S. Gradshteyn and I. M. Ryzhik, Table of Integrals, Series, and Products, Academic Press, Boston, Mass, USA, 5th edition, 1994.

[12] M. Abramowitz and I. A. Stegun, Eds., Handbook of Mathematical Functions with Formulas, Graphs, and Mathematical Tables, vol. 55 of National Bureau of Standards, Applied Mathematics Series, U.S. Government Printing Office, Washington, DC, USA, 1968.

[13] http://mathworld.wolfram.com/OrthogonalPolynomials.html.

[14] W. Magnus and F. Oberhettinger, Formeln und Sätze für die speziellen Funktionen der mathematischen Physik, Springer, Berlin, Germany, 2nd edition, 1948.

[15] G. Szëgo, Orthogonal Polynomials, vol. 23 of AMS Colloquium Publications, American Mathematical Society, Providence, RI, USA, 1939.

[16] F. Marcellán and R. Álvarez-Nodarse, "On the "Favard theorem" and its extensions," Journal of Computational and Applied Mathematics, vol. 127, no. 1-2, pp. 231-254, 2001.

[17] D. J. Dickinson, H. O. Pollak, and G. H. Wannier, "On a class of polynomials orthogonal over a denumerable set," Pacific Journal of Mathematics, vol. 6, pp. 239-247, 1956.

[18] M. Bruschi, F. Calogero, and R. Droghei, "Polynomials defined by three-term recursion relations and satisfying a second recursion relation: connection with discrete integrability, remarkable (often Diophantine) factorizations," In preparation. 


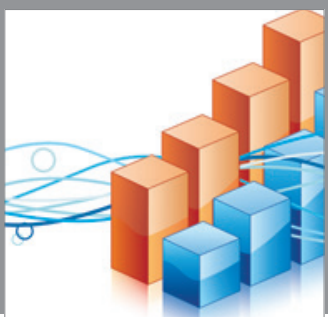

Advances in

Operations Research

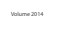

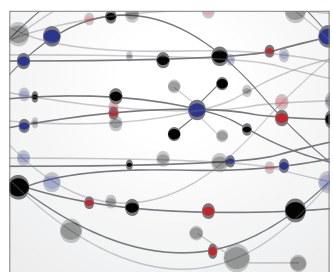

\section{The Scientific} World Journal
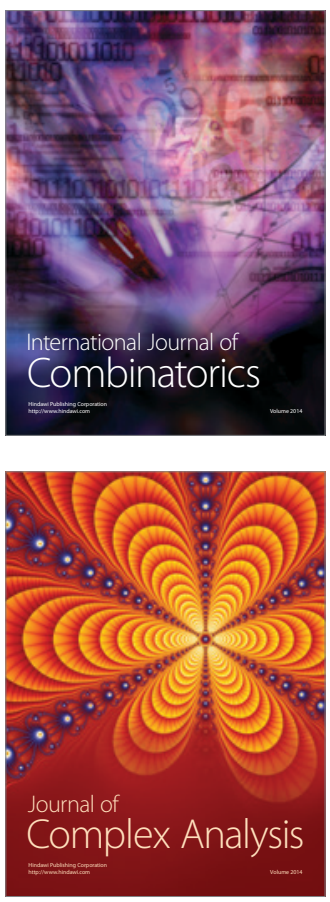

International Journal of

Mathematics and

Mathematical

Sciences
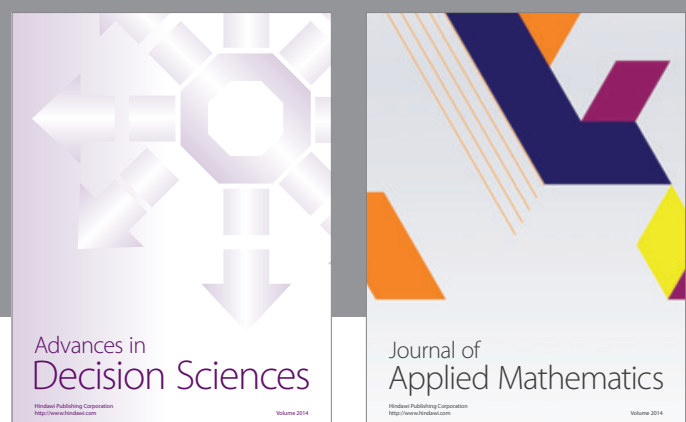

Journal of

Applied Mathematics
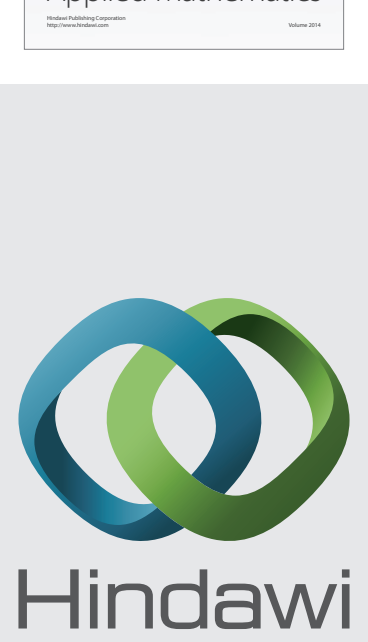

Submit your manuscripts at http://www.hindawi.com
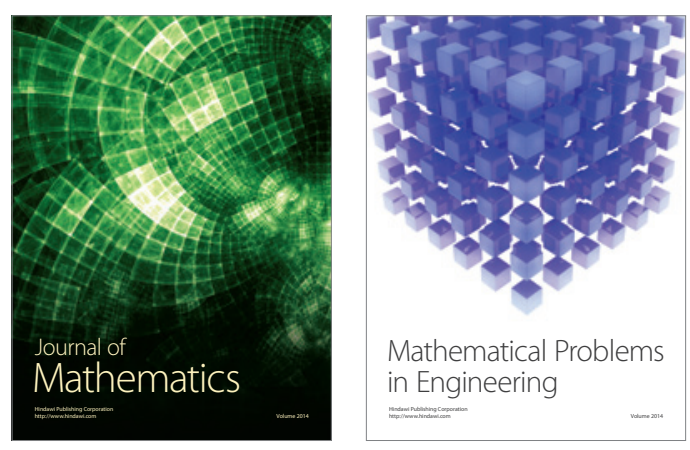

Mathematical Problems in Engineering
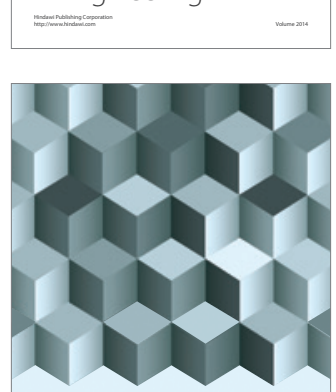

Journal of

Function Spaces
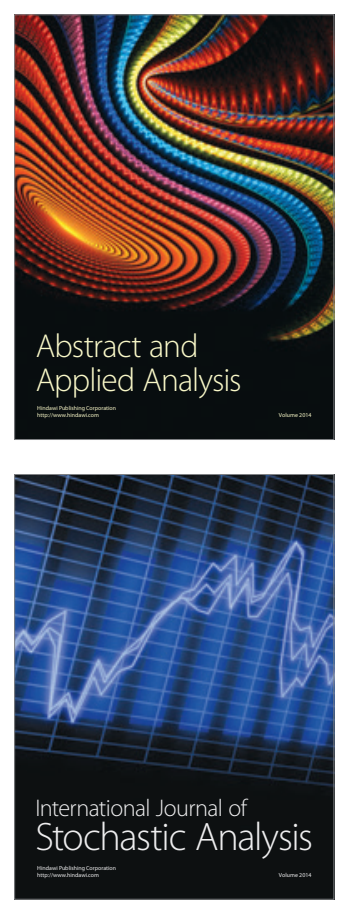

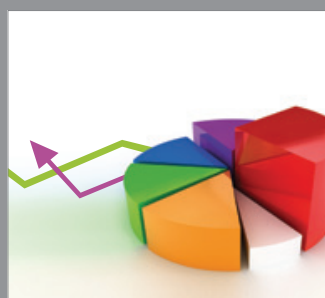

ournal of

Probability and Statistics

Promensencen
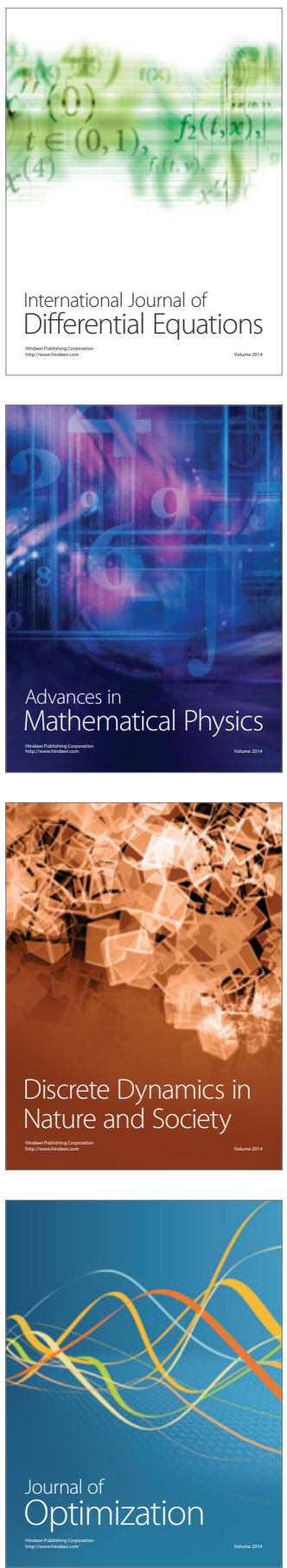